\title{
Flow Between Two Sites on a Percolation Cluster
}

\author{
José S. Andrade Jr. ${ }^{1,2}$, Sergey V. Buldyrev ${ }^{1}$, Nikolay V. Dokholyan ${ }^{1,3}$, Shlomo Havlin ${ }^{1,4}$, \\ Peter R. King ${ }^{5}$, Youngki Lee ${ }^{1}$, Gerald Paul ${ }^{1}$, and H. Eugene Stanley ${ }^{1}$ \\ ${ }^{1}$ Center for Polymer Studies, Boston University, Boston, MA 02215 \\ ${ }^{2}$ Departamento de Fúsica, Universidade Federal do Ceará, 60451-970 Fortaleza, Ceará, Brazil \\ ${ }^{3}$ Department of Chemistry and Chemical Biology, Harvard University \\ 12 Oxford Street, Cambridge, MA 02138, USA \\ ${ }^{4}$ Minerva Center 83 Department of Physics, Bar-Ilan University, Ramat Gan, Israel \\ ${ }^{5}$ Centre for Petroleum Studies, TH Huxley School, Imperial College \\ Prince Consort Road, London SW7 2BP, UK
}

(Submitted: 21 April 2000)

\begin{abstract}
We study the flow of fluid in porous media in dimensions $d=2$ and 3 . The medium is modeled by bond percolation on a lattice of $L^{d}$ sites, while the flow front is modeled by tracer particles driven by a pressure difference between two fixed sites ("wells") separated by Euclidean distance $r$. We investigate the distribution function of the shortest path connecting the two sites, and propose a scaling Ansatz that accounts for the dependence of this distribution (i) on the size of the system, $L$, and (ii) on the bond occupancy probability, $p$. We confirm by extensive simulations that the Ansatz holds for $d=2$ and 3 , and calculate the relevant scaling parameters. We also study two dynamical quantities: the minimal traveling time of a tracer particle between the wells and the length of the path corresponding to the minimal traveling time "fastest path", which is not identical to the shortest path. A scaling Ansatz for these dynamical quantities also includes the effect of finite system size $L$ and off-critical bond occupation probability $p$. We find that the scaling form for the distribution functions for these dynamical quantities for $d=2$ and 3 is similar to that for the shortest path but with different critical exponents. The scaling form is represented as the product of a power law and three exponential cutoff functions. We summarize our results in a table which contains estimates for all parameters which characterize the scaling form for the shortest path and the minimal traveling time in 2 and 3 dimensions; these parameters are the fractal dimension, the power law exponent, and the constants and exponents that characterize the exponential cutoff functions.
\end{abstract}

\section{INTRODUCTION}

Percolation theory is a paradigmatic model for connectivity, originally introduced as a mathematical subject in the late 1950s [1]. Thereafter, percolation theory has been found useful to characterize many disordered systems [2] 6 .

The simplest percolation model is a lattice of bonds occupied with probability $p$. Neighboring bonds are considered to be connected if both are occupied. A set of sites connected by bonds is called a cluster. As $p$ increases, new clusters are formed and previously existing clusters not only grow, but become connected as more sites are occupied in the system. At a critical value of $p, p_{c}$ (known as the percolation threshold), one spanning cluster appears and provides overall connectivity. Just at the critical point, the incipient infinite percolation cluster is an example of a random fractal that is a useful model for real disordered systems. While the actual threshold value $p_{c}$ depends on the particular lattice chosen, the behavior of the properties measured near the percolation threshold is universal, depending only on the dimensionality of the system. For example, the mass $M$ of a cluster diverges at the percolation threshold as a power $M \sim\left|p-p_{c}\right|^{-\gamma}$ where $\gamma=43 / 18(d=2)$ and $1.795 \pm 0.005$ $(d=3)$ regardless of the type of lattice 2 , 4 . This universality concept is extremely useful as it means that we can understand a new system knowing that its critical exponents are the same as previously-studied systems. Also, off-lattice continuum percolation appears to have the same exponent values as those for lattice percolation [8.9.

A comprehensive set of exactly- and numericallycalculated critical exponents is now available to describe many of the features of percolation [2, 4 , and the percolation paradigm has been applied to problems of practical interest in heterogeneous chemistry [7], polymer science 10] and transport phenomena in disordered systems [11. In studying transport phenomena in disordered systems, one must couple additional processes to the geometrical features of the percolation representation. Typical examples include the problems of diffusion, reaction and flow through porous media, as well as the metalto-insulator transition in polymer composites and alloys. These are systems in which the interplay between structure and phenomenology must be investigated in detail, as it might be the relevant factor determining optimum material properties.

A useful concept - first put forward by Ambegaokar, Halperin and Langer [12] - is that electrical transport in 
disordered media with a broad distribution of conductance values is dominated by those regions where the conductances are larger than some critical value $\sigma_{c}$. This critical value is the largest conductance such that the set of conductances above this threshold value still preserves the global connectivity of the system. In percolation terminology, this cluster would be analogous to the conducting spanning cluster. It is then possible to reduce the general problem of transport in a highly connected disordered media with a broad distribution of conductances to a percolation problem at criticality. Once the "critical cluster" is identified within the disordered geometry, one can estimate macroscopic transport properties of the system. This approximation, commonly called "the critical path method" [6], has been extended by Katz and Thompson 13 to estimate transport properties (e.g., permeability and electrical conductivity) in disordered materials. Thus, although the majority of studies of fluid flow through disordered media are for systems well above the threshold (e.g., sandstone), if the distribution of permeabilities is sufficiently broad, we can still make use of percolation concepts to model the relevant geometry of well-connected disordered media. Therefore, percolation theory is certainly an appropriate description for a large number of disordered systems (see also Ref. 114 and references therein). This is the basis of our study and also the basis of the vast literature on fluid flow in percolation clusters.

The aim of the present paper is to discuss the potential application of percolation theory as a convenient geometrical model for understanding numerous aspects of flow through porous rocks 15,16]. Special emphasis will be given to the study of oil displacement, i.e., how hydrocarbons propagate through geological formations between a pair of wells in the oil field. This work could also be applied to the breakthrough time for contamination of a water supply, or the time for released radioactive material to get from a leaking nuclear repository into the biosphere.

Oil fields are extremely complex, containing geological heterogeneities on a wide range of length scales from centimeters to kilometers. These heterogeneities, caused by the sedimentary processes that deposited the rocks and the subsequent actions on the rock, such as fracturing by tectonic forces and mineral deposition from aquifer flow, have a significant impact on hydrocarbon recovery.

The most common method of oil recovery is by displacement. Either water or a miscible gas (carbon dioxide or methane) is injected in a well (or wells) to displace the oil to other wells. Ultimately the injected fluid will break through into a production well where it must be separated from the oil, which is a very costly process. Once the injected fluid has broken through, the rate of oil production declines as more injected fluid is produced. For economic purposes it is important to know when the injected fluid will break through and what the rate of decline of oil production will be so that the economic limit of production can be determined.
Because the sedimentary process that produces the porous rock through which the fluid flows is very chaotic, the rock is highly heterogeneous. However, in many cases the rock can be separated into two types - high permeability ("good") and low or zero permeability ("bad") and for all practical purposes we can assume the flow takes place only in the good rock. The spatial distribution of the rock types is often close to random, in which case the classical percolation problem is a good approximation. The place of the occupancy probability $p$ is taken by the volume fraction of the good rock, called the net-to-gross ratio in the petroleum literature.

We have very little direct knowledge about the spatial distribution of rock properties in a reservoir. Direct measurements are limited to samples that represent a fraction of $\approx 10^{-13}$ of the total reservoir volume. These samples are taken from the well locations. Elsewhere the properties have to be inferred from knowledge of the general geological environment and by analogy with other reservoirs or surface outcrops. Hence, there is a great deal of uncertainty in our prediction of the spatial distribution of these rock properties. This leads to an uncertainty in our ability to predict the flow performance, principally the breakthrough time and the production decline rate. We need to estimate the uncertainty accurately so that economic risk evaluations can be made.

The conventional approach to this problem is to build a detailed (numerical) model of the rock properties. These models will honor the one and two point statistics observed from the wells and analogue outcrops. The models must also agree with the observed data values at wells. These models are statistical in nature and conventionally one samples realizations from the models and performs numerical flow calculations on the realizations to give a Monte Carlo prediction of breakthrough and production decline. Unfortunately this process is so time consuming as to be impractical in many cases. Typically the flow simulation can take several hours on reasonable workstations. When hundreds of realizations are sampled to get good statistics, the total computing time becomes unwieldy. Thus there is a strong need to make this more efficient so as to come up with very quick, but accurate, predictions of recovery and the uncertainty due to the lack of knowledge of the underlying rock properties.

The purpose of this work is to accomplish the goals described above using methods derived from percolation theory. It is based on two key assumptions. One is that for many cases the permeability disorder can be approximated by either permeable or impermeable rock. For example the reservoir may have been deposited by meandering river belts in which case the good sand occurs as "packages" in an insulating background. The other assumption is that the flow paths are strongly controlled by the permeability disorder and not strongly modified by the flow dynamics themselves. Again there are many cases when this is reasonable, in particular if the viscosity ratio between the injected and displaced fluids is not too large or when the system is highly disordered. 
Under these assumptions we can consider the underlying heterogeneity to be that of a percolating system (not necessarily at threshold). This has previously been done to study the fraction of sand connected to well pairs [17]. We then look at the dynamic displacement on this cluster where the flow is controlled by Darcy's law (analogous to Ohm's law in electrical current flow). We assume that the injected fluid can be treated as a passive tracer (i.e., one that is not absorbed by the rock) that is convected along these flow paths. Then the breakthrough time is the same as the first passage time and is strongly controlled by the shortest path length between the wells; the post breakthrough production decline is controlled by the longer paths. Figure 11a illustrates a typical percolation backbone and the shortest path between two points. Note that the lines here represent not microscopic pores but rather sand bodies whose size are of the order of tens of meters.

The rest of the paper is organized as follows. In Sec. II, because of its importance to flow in the cluster, we study the distribution of shortest paths between two sites in a percolation cluster. In Sec. III we study the distribution functions for the dynamic quantities: minimal traveling time, and length of the path corresponding to minimal traveling time. Finally, in Sec. IV, we draw some conclusions and discuss possible future work.

\section{SHORTEST PATH}

This section deals with the distribution of the shortest path between two sites on a percolation cluster. Because of the qualitative resemblance between the shortest path and the minimal traveling time of a tracer particle, the first step in understanding fluid transport between two sites in a percolation system is to characterize the geometrical properties of the shortest connecting path. For example, if we assume that the traveling time along a path is proportional to the path length (i.e., all velocities are equal), then we can obtain a rough estimate for the traveling time from purely geometrical arguments.

\section{A. Basic distribution functions}

The shortest path or chemical distance, $\ell$, between two sites on a percolation cluster is defined as the shortest path connecting the two sites (Fig. 1) [18,19]. The typical value $\ell^{*}$ of the shortest path between two sites on a cluster scales with the geometrical distance, $r$, between these points as

$$
\ell^{*} \sim r^{d_{\min }}
$$

where

$$
d_{\min }= \begin{cases}1.13 \pm 0.02 & {[d=2]} \\ 1.374 \pm 0.02 & {[d=3]}\end{cases}
$$

is the fractal dimension of the shortest path [20,21].

Consider a hypercubic lattice of $L^{d}$ sites. All information about the distribution of shortest paths is contained in the joint probability density function $P(r, \ell)$, i.e., the probability that two sites on the same spanning cluster are separated by geometrical distance $r$ and chemical path $\ell$. We sum over all chemical paths $\ell$ to calculate the probability distribution that the Euclidean distance between two sites is $r$,

$$
P(r) \equiv \int P(r, \ell) d \ell .
$$

Similarly, we obtain the probability distribution that two sites are separated by the chemical distance $\ell$ by summing over all possible geometrical distances,

$$
P(\ell) \equiv \int P(r, \ell) d r
$$

Given that the shortest distance between these sites is $\ell$, the conditional probability that the geometrical distance between two sites is $r$ is

$$
P(r \mid \ell)=\frac{P(r, \ell)}{P(\ell)} .
$$

For isotropic media this function has been studied extensively and $P(r \mid \ell)$ is of the form [3, 22, 24]

$$
P(r \mid \ell)=A_{\ell}\left(\frac{r}{\ell^{\tilde{\nu}}}\right)^{g_{r}} \exp \left(-a\left(\frac{r}{\ell^{\tilde{\nu}}}\right)^{\tilde{\delta}}\right),
$$

where

$$
\tilde{\delta}=\frac{1}{1-\tilde{\nu}}=\frac{d_{\min }}{d_{\min }-1}
$$

and

$$
\tilde{\nu} \equiv 1 / d_{\min }
$$

For $d=2$, Ziff recently argued [25] that

$$
g_{r}-1=25 / 24 \quad[d=2] .
$$

Our simulations confirm the analytical form of the $P(r \mid \ell)$ as well as these values of $\tilde{\nu}$ and $g_{r}$ (see Fig. 2a).

The function of interest to us is the conditional probability for two sites to be separated by the shortest path $\ell$, given that the geometrical distance between these sites is $r$

$$
P(\ell \mid r)=\frac{P(r, \ell)}{P(r)} .
$$

¿From (10) and (5), we see that $P(r \mid \ell)$ and $P(\ell \mid r)$ are related

$$
P(\ell \mid r)=P(r \mid \ell) \frac{P(\ell)}{P(r)}
$$


At the percolation threshold, it has been shown 15 that, in analogy with (6),

$$
P(\ell \mid r) \sim \frac{1}{r^{d_{\min }}}\left(\frac{\ell}{r^{d_{\min }}}\right)^{-g_{\ell}} \exp \left(-a\left(\frac{\ell}{r^{d_{\min }}}\right)^{-\phi_{\ell}}\right)
$$

where

$$
\begin{gathered}
g_{\ell}-1=\frac{\left(g_{r}-1\right)+\left(2-d_{f}\right)}{d_{\min }}, \\
\phi_{\ell}=\tilde{\delta} \tilde{\nu}=\tilde{\nu} /(1-\tilde{\nu})=\frac{1}{d_{\min }-1},
\end{gathered}
$$

and

$$
d_{f}= \begin{cases}91 / 48 & {[d=2]} \\ 2.524 \pm 0.008 & {[d=3]}\end{cases}
$$

is the fractal dimension of the incipient infinite cluster [1]. Substituting (9) into (13), we find for $d=2$

$$
g_{\ell}=2.01 \pm 0.02 \quad[d=2] .
$$

The probability distribution of more practical interest is $P^{\prime}(\ell \mid r)$, defined in the same way as $P(\ell \mid r)$ but for any two randomly-chosen points separated by geometrical distance $r$ and on the same cluster, but not necessarily on the incipient infinite cluster 15]. As shown in Appendix A, $P^{\prime}(\ell \mid r)$ has the same scaling form as in Eq. (12), but with $g_{\ell}$ replaced by

$$
g_{\ell}^{\prime}=g_{\ell}+\frac{d-d_{f}}{d_{\min }} .
$$

Figure 2 $2 \mathrm{~b}$ illustrates the difference between $g_{\ell}$ and $g_{\ell}^{\prime}$.

\section{B. Distribution of shortest path}

The complete scaling form of $P^{\prime}(\ell \mid r)$, which accounts also for finite size effects and off-critical behavior, has been studied for $d=2$ and reported in 15 . Specifically, the following Ansatz has been proposed [15]

$$
\begin{aligned}
P^{\prime}(\ell \mid r) \sim & \frac{1}{r^{d_{\min }}}\left(\frac{\ell}{r^{d_{\min }}}\right)^{-g_{\ell}^{\prime}} f_{1}\left(\frac{\ell}{r^{d_{\min }}}\right) . \\
& f_{2}\left(\frac{\ell}{L^{d_{\min }}}\right) f_{3}\left(\frac{\ell}{\xi^{d_{\min }}}\right)
\end{aligned}
$$

where $\xi \sim\left|p-p_{c}\right|^{-\nu}$ is the pair connectedness length, and the scaling functions have the form

$$
\begin{gathered}
f_{1}(x) \equiv \exp \left(-a x^{-\phi}\right), \\
f_{2}(x) \equiv \exp \left(-b x^{\psi}\right),
\end{gathered}
$$

and

$$
f_{3}(x) \equiv \exp (-c x)
$$

The function $f_{1}$ accounts for the lower cut-off due to the constraint $\ell>r$, while $f_{2}$ and $f_{3}$ account for the upper cut-offs due to the finite size effect and due to the finite correlation length respectively. Either $f_{2}$ or $f_{3}$ becomes irrelevant, depending on the magnitudes of $L$ and $\xi$ : for $L<\xi, f_{2}$ dominates the upper cut-off, otherwise $f_{3}$ dominates. We assume the independence of the finite size effect and the effect of the concentration of the occupied sites, so that Eq. (18) can be represented as a product of the terms which are responsible for the finite size effect $\left(f_{2}\right)$ and the effect of the concentration $\left(f_{3}\right)$. Simulations for $d=2$ [15 have been used to test this assumption.

\section{Shortest path in three dimensions}

\section{Behavior at criticality}

Here we extend the study of $P^{\prime}(\ell \mid r)$ to $d=3$. We numerically test the scaling conjecture (18) exactly at the percolation threshold $p=p_{c}$-in which case $\xi=\infty$ so $f_{3}=f(0)=$ const. We build clusters using the Leath algorithm 18, 19,26]. Since the Leath algorithm corresponds to the process of selecting a random point on the lattice, the probability $P^{\prime}(\ell \mid r)$ is equal to the probability that a pair of randomly selected points has chemical distance $\ell$ and geometrical distance $r$, given that they belong to the same cluster, a cluster that is not necessarily the infinite cluster. Hence Eq. (18) reduces to

$$
\begin{aligned}
P^{\prime}(\ell \mid r) \sim & \frac{1}{r^{d_{\min }}}\left(\frac{\ell}{r^{d_{\min }}}\right)^{-g_{\ell}^{\prime}} \cdot \\
& f_{1}\left(\frac{\ell}{r^{d_{\min }}}\right) f_{2}\left(\frac{\ell}{L^{d_{\min }}}\right) \quad\left[p=p_{c}\right] .
\end{aligned}
$$

Figure 3a shows that, in the range $r^{d_{\min }}<\ell<L^{d_{\min }}$, $P^{\prime}(\ell \mid r)$ has power-law behavior with slope

$$
g_{\ell}^{\prime}=2.3 \pm 0.1, \quad[d=3]
$$

and rapidly vanishes for $\ell<r^{d_{\min }}$ and for $\ell>L^{d_{\min }}$. To determine the functions $f_{1}$ and $f_{2}$, we compute the rescaled probability distribution

$$
\Phi\left(\frac{\ell}{r^{d_{\min }}}\right) \equiv P^{\prime}(\ell \mid r) \ell^{g_{\ell}^{\prime}} r^{-d_{\min }\left(g_{\ell}-1\right)},
$$

and plot it against scaling variable $x \equiv \ell / r^{d_{\text {min }}}$ (see Fig. 3b) using the value $d_{\min }=1.374$. According to Eq. (22)

$$
\Phi(x)=A f_{1}(x) f_{2}\left[x\left(\frac{r}{L}\right)^{d_{\min }}\right] .
$$


Therefore, $\Phi(x)$ should depend only on $x$ and the ratio $r / L$. Indeed, Fig. 3 $\mathrm{b}$ shows excellent data collapse for $L / r=8$, with sharp cutoffs governed for $x<1$ by $f_{1}(x)$ and for $x>(L / r)^{d_{\min }}$ by $f_{2}\left[x(r / L)^{d_{\min }}\right]$.

In order to test the assumption that the functions $f_{1}$ and $f_{2}$ are stretched exponentials with exponents $\phi_{\ell}$ and $\psi_{\ell}$, we plot

$$
\Pi(x) \equiv \log _{10}[A / \Phi(x)]
$$

versus $x$ in double logarithmic scale for various values of normalization constant $A$ (see Fig. 3ic). If the stretched exponential conjecture is correct, $\Pi(x)$ should have two straight line asymptotes for $\log x \rightarrow+\infty$ with the slope $\psi_{\ell}$ and for $\log x \rightarrow-\infty$ with the slope $-\phi_{\ell}$. We find that the slopes $\phi_{\ell}$ and $\psi_{t_{\min }}$ of the straight line fits depend weakly on the value of $A$. Using $A=0.08$, we obtain the longest regimes of straight line behavior. For this value of $A$, we find $\phi_{\ell} \approx 2.1$ and $\psi_{\ell} \approx 2.5$. Equation (14) yields a predicted value of $\phi_{\ell}=2.67$ in good agreement with our simulation result.

\section{Off-critical behavior}

For $p \neq p_{c}$, we identify three regimes determined by the value of the connectedness length, $\xi$, in relation to the values of $r$ and $L$ :

(i) $\xi>L>r$. In this regime, the fact that $p \neq p_{c}$ cannot be detected because the connectedness length is larger than the other relevant variables.

(ii) $L>\xi>r$. In this case, the upper cutoff of the distribution Eq. (18) is governed by $f_{3}$ and the functional form of the rescaled probability $\Phi$ is given by

$$
\Phi\left(\ell / r^{d_{\min }}\right) \sim f_{1}\left(\frac{\ell}{r^{d_{\min }}}\right) f_{3}\left(\frac{\ell}{\xi^{d_{\min }}}\right) .
$$

For large $\ell$, we suggest an exponential decay of $\Phi$

$$
\Phi\left(\ell / r^{d_{\min }}\right) \sim \exp \left(-c \frac{\ell}{\xi^{d_{\min }}}\right) .
$$

Indeed, for $p<p_{c}$, semi-logarithmic plots of $\log \Phi\left(\ell / r^{d} \min \right)$ versus $\ell$ shown in Fig. 国a can be approximated by straight lines with slopes which approach zero as $p \rightarrow p_{c}$. According to Eq. (28), these slopes $k(p)$ should be proportional to $\xi^{-d_{\min }} \sim$ $\left|p-p_{c}\right|^{d_{\min } \nu} \approx\left|p-p_{c}\right|^{1.19}$. Figure $\llbracket \mathrm{b}$ shows a double logarithmic plot of $|k(p)|$ versus $\left|p-p_{c}\right|$ for $p<p_{c}$. This curve can be well approximated by a straight line with slope 1.22 in good agreement with the scaling conjecture (22). For $p>p_{c}$ a similar analysis should hold. However, limitations on the size of the system we can simulate make the analysis problematic. Figure 4 c shows $P^{\prime}(\ell)$ for various values of $p>p_{c}$. Note that it is only for values of $p \geq p_{c}+0.03$ that the distributions "cut-off" at smaller $\ell$ than the distribution for $p=p_{c}$. Thus it is only for values of $p-p_{c} \geq 0.03$ that the large $\ell$ behavior of Eq. (18) is determined by the fact that the system is not at criticality (i.e., by $f_{3}$ ) as opposed to being determined by the finite size of the system (i.e., by $f_{2}$ ). Below $p=p_{c}+0.03, \xi$ is still greater than $L$. On the other hand, if $p$ is not close to $p_{c}$, the scaling form is not expected to hold. Thus, the results are inconclusive based on the sizes of the systems we can generate - we cannot determine the parameters that govern the large $\ell$ behavior of Eq. (18) above $p_{c}$.

(iii) $L>r>\xi$. When the connectedness length $\xi$ is smaller than the distance $r$ between the wells, the system can be considered homogeneous [28,27]. This can be seen in Fig. Fa in which we plot $P(\ell \mid r)$ for various values of $r$ at $p=0.7$ for $2 \mathrm{~d}$ site percolation $\left(p_{c}=0.593\right)$. As $r$ increases from below to above the connectedness length, the form of the distribution changes from the power law distribution of Eq. (18) to a Gaussian distribution with a pronounced peak, a characteristic of homogeneous systems. Furthermore, as shown in Fig. 5b, the fractal dimension of the shortest length crosses over from $d_{\min }=1.13$ to $d_{\min }=1.0$, characteristic of a homogeneous system [28,27]. The convergence to a Gaussian can be expected due to the following considerations. The minimal path connecting the wells separated by distance $r$ passes through $r / \xi$ independent blobs. For each of these blobs, the probability distribution for the shortest path across the blob, $\ell_{b}$, is still given by Eq. (18), but with $r$ and $L$ replaced by $\xi$ and $\ell$ replaced by $\ell_{b}$. This distribution is characterized by $\left\langle\ell_{b}\right\rangle \sim \xi^{d}$ min and variance $\sigma_{b}^{2} \equiv\left\langle\ell_{b}^{2}\right\rangle-\left\langle\ell_{b}\right\rangle^{2} \sim \xi^{2 d_{\text {min }}}$. The total minimal path is the sum of $n=r / \xi$ independent variables $\ell_{b}$, hence it converges to a Gaussian with

$$
\langle\ell\rangle \sim r \xi^{d_{\min ^{-1}}} \quad \text { and } \quad \sigma^{2} \sim r \xi^{2 d_{\min }-1} .
$$

Thus the slope of the graph, $k(p)$, of $\langle\ell\rangle$ vs $r$ in Fig. 5c should decay as

$$
k(p) \sim\left|p-p_{c}\right|^{-\nu\left(d_{\min }-1\right)}=\left|p-p_{c}\right|^{-0.17}
$$

and the slope of $\sigma^{2}$ versus $r$ should decay as

$$
\left|p-p_{c}\right|^{-\nu\left(2 d_{\min }-1\right)}=\left|p-p_{c}\right|^{-1.7} .
$$

Indeed, (see Fig 5 d) we see that the slope of $\sigma^{2}$ versus $r$ decays with $p$ more strongly than that of $\langle\ell\rangle$ versus $r$. The numerical values of slopes from Figs. 5.c and 5 $\mathrm{d}$ are in good agreement with the theoretical predictions Eqs. (30,31). For $d=3$ we expect similar behavior. 


\section{Rectangular boundary conditions}

Since realistic oil fields do not have square boundaries, it is reasonable to ask what is the effect of rectangular boundary conditions. Here we study the distributions of the shortest length and minimal time on a twodimensional lattice of size $L_{x} \times L_{y}, L_{x} \neq L_{y}$. We position the wells at points $A$ and $B$ separated by distance $r=16$ along $x$-axis. We study two cases (Fig. 6):

(a) $L_{x}$ is fixed $\left(L_{x}=32\right)$, and we vary $L_{y}\left(L_{y}=64\right.$, $128,256,512,1024)$

(b) $L_{y}$ is fixed $\left(L_{y}=32\right)$, and we vary $L_{x}\left(L_{x}=64\right.$, $128,256,512,1024)$.

We find that (i) the shortest length and minimal time distributions are identical in all of the above cases and (ii) the scaling form for these anisotropic cases is the same as the isotropic case with $L$ replaced by the minimum of $L_{x}$ and $L_{y}$, i. e. the scaling form of the distribution Eq. (18) remains unchanged with the exception that $L$ is determined by

$$
L=\min \left(L_{x}, L_{y}\right) .
$$

Equation (32) can be a result of competing exponentials $f_{2 x}$ and $f_{2 y}$. Since both $f_{2 x}$ and $f_{2 y}$ are rapidly decaying functions with $L_{x}$ and $L_{y}$ [see Eq. (20)], the finite-size cutoff of $P(\ell \mid r)$ is determined by the smaller of $L_{x}$ and $L_{y}$. The fact that the results are independent of the axis along which the wells are aligned can be understood by realizing that the probability that "oblong" clusters connect two points separated by a distance longer than the minimum of $L_{x}$ and $L_{y}$ is low. This finding has the implication that in anisotropic fields, a number of well pairs would be needed to optimize the recovery of oil in the field.

\section{MINIMAL TRAVELING TIME AND FASTEST PATH}

We turn next to dynamics, the study of flow on percolation clusters, which has close ties to such applications as hydrocarbon recovery and ground-water pollution [429 32]. In this section, we discuss the properties of the flow on $d=2$ and $d=3$ bond percolation clusters. Specifically, we investigate the scaling properties of the distributions of minimal traveling time and the length of the path corresponding to the minimal traveling time (fastest path) of the tracer particles. Some of the results in $d=2$ were reported previously [16]. Here we extend the work to $d=3$, and study the effects of finite system size and off-criticality for $d=2$ and $d=3$.

\section{A. The model}

We study incompressible flow between two sites $A$ and $B$ separated by Euclidean distance $r$ (see Fig. 1). To model the flow front, we use passive tracers - particles not absorbed by the surroundings, that move only by convection, ignoring molecular diffusion which is slow on the time scales of interest. The convection is governed by the flow field due to the pressure difference between sites connected by the bonds. We simulate the flow of a tracer particle starting from the injection point $A$ traveling through the medium along a path connected to the recovery point $B$. The dynamics of flow at a macroscopic level on the percolation cluster is determined by the local flow (local currents) on the individual bonds in the backbone of the cluster. The velocity of a tracer at each bond is determined by the pressure difference across that bond (Darcy's law [33]):

$$
v_{i j}=T\left(P_{j}-P_{i}\right),
$$

where $P_{i}$ and $P_{j}$ are the values of pressure at sites $i$ and $j$. The coefficient $T$, which is a function of permeability $k$, viscosity $\eta$, and the length of a bond $L_{b}\left(T=k /\left(\eta L_{b}\right)\right)$, is set to 1 . We normalize the velocities assuming the total flow $J$ between $A$ and $B$ is fixed, independent of the distance between $A$ and $B$, and the realization of the porous media. This resembles more closely oil recovery processes where constant flow, as opposed to constant pressure, is maintained.

We obtain the pressure difference across each bond by solving Kirchhoff's law

$$
\sum_{j} v_{i j}=0
$$

for each node $i$ in the cluster where the summation is over all bonds connected to that node. Fig. 1 $1 \mathrm{~b}$ shows the results of solving these equations for the cluster discussed in Section I (Fig. 1 b). Magnitudes of currents on cluster backbone are depicted in gray scale with the lightest areas corresponding to the smallest currents and the darkest to the largest currents.

We define the traveling time, $\tilde{t}$, of a path $\mathcal{C}$ as the sum of the tracer's traveling times $t_{i j}$ at each bond $(i j)$ joining sites $i$ and $j$ which are on the path,

$$
\tilde{t}=\sum_{(i j) \in \mathcal{C}} t_{i j}
$$

The traveling length, $\tilde{\ell}$, in turn, is the number of bonds present in path $\mathcal{C}$. Among the ensemble of all paths $\{\mathcal{C}\}$, we select the path $\mathcal{C}^{*}$ that has the minimal traveling time, $t_{\min }$

$$
t_{\min }\left(\mathcal{C}^{*}\right)=\min _{\{\mathbf{C}\}} \tilde{t}(\mathcal{C})
$$

and we define the length of the fastest path, $\ell_{\min }$, corresponding to the minimal traveling time, as the number of bonds present in path $\mathcal{C}^{*}$. The first quantity $t_{\min }$ is 
the breakthrough time of the gas/liquid that displaces the oil during recovery and has fundamental importance to the oil industry. The quantity $\tilde{t}$ determines postbreakthrough behavior. We also define the exponents $d_{x}$, where $x$ denotes $\ell_{\min }, t_{\min }, \tilde{\ell}$ or $\tilde{t}$ by

$$
x^{*} \sim r^{d_{x}}
$$

and where $x^{*}$ is the characteristic (most probable) length or time of the corresponding distribution.

Using a "burning" algorithm [34], we then find the minimal time and the fastest path for the particle to travel between points $A$ and $B$. Figure 1 1 shows the propagation of the tracer particles through the same backbone shown in Fig. 1a and 1 $1 \mathrm{~b}$. At $t=t_{\min }$, the tracer particles spread over $t=t_{\min } \cdot J$ bonds, which constitute a subset of the backbone with fractal dimension $d_{\mathrm{tm}}$, which is larger than the fractal dimension of the minimal path but smaller than the fractal dimension of the entire backbone $d_{\mathrm{B}}$. Hence

$$
d_{\mathrm{min}}<d_{\mathrm{tm}}<d_{\mathrm{B}}
$$

\section{B. Minimal Traveling Time}

We first study the minimal traveling time for $d=2$. In Fig. 7, a scatter plot of the minimal traveling time versus shortest path, we see that the minimal times are strongly correlated with the shortest paths in the realizations simulated, $t_{\min } \sim \ell^{z}$, where $z \approx 1.17$. Since $\ell$ scales as $r^{d_{\text {min }}}$ we propose that $t_{\min }$ scales as $r^{d_{\mathrm{tm}}}$ with $d_{\mathrm{tm}}=z d_{\min }=1.33$. This suggests that the same scaling form which applies to the distribution of shortest paths can also be applied to the distribution of minimal times, but with different exponents and amplitudes. Thus, we expect the Ansatz similar to Eq. (18)

$$
\begin{aligned}
P^{\prime}\left(t_{\mathrm{min}} \mid r\right) \sim & \frac{1}{r^{d_{\mathrm{tm}}}}\left(\frac{t_{\mathrm{min}}}{r^{d_{\mathrm{tm}}}}\right)^{-g_{\mathrm{tm}}^{\prime}} f_{1}\left(\frac{t_{\mathrm{min}}}{r^{d_{\mathrm{tm}}}}\right) . \\
& f_{2}\left(\frac{t_{\mathrm{min}}}{L^{d_{\mathrm{tm}}}}\right) f_{3}\left(\frac{t_{\mathrm{min}}}{\xi^{d_{\mathrm{tm}}}}\right),
\end{aligned}
$$

where the scaling functions are $f_{1}(x)=\exp \left(-a_{\mathrm{tm}} x^{-\phi_{\mathrm{tm}}}\right)$, $f_{2}(x)=\exp \left(-b_{\mathrm{tm}} x^{\psi_{\mathrm{tm}}}\right)$ and $f_{3}(x)=\exp \left(-c_{\mathrm{tm}} x^{\pi_{\mathrm{tm}}}\right)$. Here $\xi$ is a characteristic length of the pair connectedness function and has a power-law dependence on the occupancy probability $p$ as

$$
\xi \sim\left|p-p_{c}\right|^{-\nu} .
$$

The first function $f_{1}$ accounts for the lower cut-off due to the constraint $\ell>r$, while $f_{2}$ and $f_{3}$ account for the upper cut-offs due to the finite size effect and due to the finite connectedness length, respectively. Either $f_{2}$ and $f_{3}$ becomes irrelevant, depending on which of the two values $L$ or $\xi$ is greater. For $L<\xi, f_{2}$ dominates the upper cut-off, otherwise $f_{3}$ dominates. We have assumed independence of the finite size effect and off-criticality effect, so that Eq. (39) can be represented as a product of the terms which are responsible for the finite size effect $\left(f_{2}\right)$ and the effect of the concentration $\left(f_{3}\right)$.

We sample over $10^{6}$ different realizations with the two sites $A$ and $B$ fixed. For each realization, we calculate exactly the minimal traveling time and the path which corresponds to the minimal traveling time to obtain $P\left(t_{\min }\right)$ and $P\left(\ell_{\min }\right)$.

\section{Behavior at criticality}

We first test numerically the scaling conjecture Eq. (39) at the percolation threshold $p=p_{c}$. In this case, $\xi=\infty$ and $f_{3}$ is a constant. Hence Eq. (39) reduces to

$$
\begin{aligned}
P^{\prime}\left(t_{\mathrm{min}} \mid r\right) \sim & \frac{1}{r^{d_{\mathrm{tm}}}}\left(\frac{t_{\mathrm{min}}}{r^{d_{\mathrm{tm}}}}\right)^{-g_{\mathrm{tm}}^{\prime}} . \\
& f_{1}\left(\frac{t_{\mathrm{min}}}{r^{d_{\mathrm{tm}}}}\right) f_{2}\left(\frac{t_{\mathrm{min}}}{L^{d_{\mathrm{tm}}}}\right) \quad\left(p=p_{c}\right) .
\end{aligned}
$$

Figure Ba shows that $P^{\prime}\left(t_{\min } \mid r\right)$ has a power-law regime with slope

$$
g_{\mathrm{tm}}^{\prime}=2.0 \pm 0.1 \text {. }
$$

To determine the functions $f_{1}$ and $f_{2}$, we compute the rescaled probability distribution

$$
\Phi\left(\frac{t_{\mathrm{min}}}{r^{d_{\mathrm{tm}}}}\right) \equiv P^{\prime}\left(t_{\mathrm{min}} \mid r\right)\left(t_{\mathrm{min}}\right)^{g_{\mathrm{tm}}^{\prime}} r^{-d_{\mathrm{tm}}\left(g_{\mathrm{tm}}-1\right)},
$$

and plot it against scaling variable $x=t_{\mathrm{min}} / r^{d_{\mathrm{tm}}}$ (see Fig. 8b). According to Eq. (41)

$$
\Phi(x)=A f_{1}(x) f_{2}\left[x\left(\frac{r}{L}\right)^{d_{\mathrm{tm}}}\right] .
$$

Therefore, $\Phi(x)$ should depend only on $x$ and the ratio $r / L$. Unlike the fractal dimension of the shortest path, $d_{\min }$, there have been no calculations of the fractal dimension of the minimal traveling time, $d_{\mathrm{tm}}$. We estimate $d_{\mathrm{tm}}$ by finding the value which yields the best data collapse for Eq. (44). For $d_{\mathrm{tm}}=1.33$, Fig. 8p shows excellent data collapse with sharp cutoffs governed for small $x<1$ by $f_{1}(x)$ and for large $x>(L / r)^{d_{\mathrm{tm}}}$ by $f_{2}\left[x(r / L)^{d_{\mathrm{tm}}}\right]$.

In order to test the assumption that the functions $f_{1}$ and $f_{2}$ are stretched exponentials with exponents $\phi_{\mathrm{tm}}$ and $\psi_{\mathrm{tm}}$, we make a log-log plot of $\Pi(x) \equiv \log _{10}[A / \Phi(x)]$ versus $x$ for various values of the normalization constant $A$ (See Fig. 8c). If the stretched exponential conjecture is correct, $\Pi(x)$ should have two straight line asymptotes for $\log x \rightarrow+\infty$ with the slope $\psi_{\mathrm{tm}}$ and for $\log x \rightarrow-\infty$ with the slope $-\phi_{\mathrm{tm}}$. The slopes $\phi_{\mathrm{tm}}$ and $\psi_{\mathrm{tm}}$ of the straight line fits depend weakly on the value of $A$. Using $A=0.14$, we obtain the longest regimes of straight 
line behavior. For this $A$ we obtained $\phi_{\mathrm{tm}} \approx 3.0$ and $\psi_{\mathrm{tm}} \approx 3.0$. With the same assumptions used to derive Eq. (14), we can derive a similar expression for $\phi_{\mathrm{tm}}$

$$
\phi_{\mathrm{tm}}=\frac{1}{d_{\mathrm{tm}}-1},
$$

which yields a predicted value of $\phi_{\mathrm{tm}}$ of 3.0 in good agreement with our simulation result.

\section{Off-Critical Behavior}

Finally, in order to test the dependence of $P^{\prime}\left(t_{\min } \mid r\right)$ on $p$ we obtain data for large system size $L(L=1000)$ and for several values of $p \neq p_{c}$. As we do for the shortest length, we analyze the behavior of $t_{\min }$ in three regimes determined by the relation of the value of the connectedness length, $\xi$, to the values of $r$ and $L$.

(i) $\xi>L>r$. In this regime, the fact that $p \neq p_{c}$ cannot be detected because the connectedness length is larger than the other relevant variables.

(ii) $L>\xi>r$. In this case, the upper cutoff of the distribution Eq. (39) is governed by $f_{3}$ and the functional form of the rescaled probability $\Phi$ is given by

$$
\Phi\left(t_{\mathrm{min}} / r^{d_{\mathrm{tm}}}\right) \sim f_{1}\left(\frac{t_{\mathrm{min}}}{r^{d_{\mathrm{tm}}}}\right) f_{3}\left(\frac{t_{\mathrm{min}}}{\xi^{d_{\mathrm{tm}}}}\right) .
$$

For large $t_{\min }$, we suggest an exponential decay of $\Phi$

$$
\Phi\left(t_{\mathrm{min}} / r^{d_{\mathrm{tm}}}\right) \sim \exp \left(-c \frac{t_{\mathrm{min}}}{\xi^{d_{\mathrm{tm}}}}\right)
$$

Semi-logarithmic plots of $\log \Phi\left(t_{\mathrm{min}} / r^{d_{\mathrm{tm}}}\right)$ versus $t_{\min }$ for $p>p_{c}$ and $p<p_{c}$ shown in Fig. $9 \mathrm{a}$ and 9b, respectively, can be approximated by straight lines with slopes which approach zero as $p \rightarrow p_{c}$. According to Eq. (47), this slope $k(p)$ should be proportional to

$$
\xi^{-d_{\mathrm{tm}}}=\left|p-p_{c}\right|^{d_{\mathrm{tm}} \nu} \approx\left|p-p_{c}\right|^{1.77} .
$$

Figure 9c shows double logarithmic plots of $|k(p)|$ versus $\left|p-p_{c}\right|$ for $p<p_{c}$ and $p>p_{c}$, which can be well approximated by straight lines with slopes 1.81 and 1.77, respectively, in good agreement with the scaling conjecture, Eq. (48). As was the case with the analysis of $P^{\prime}(\ell \mid r)>p_{c}$ for $d=3$ (see Sec. II.C.2.ii), we cannot determine the parameters that govern the large $t_{\min }$ behavior of $P^{\prime}\left(t_{\min }\right)$ because of limitations on the size of the system we can simulate. (iii) $L>r>\xi$. When the connectedness length is smaller than the distance between the wells, the behavior of the system is the same as a homogeneous system [28,27]. This can be seen in Fig. 10a in which we plot $P\left(t_{\min } \mid r\right)$ for various values of $r$ at $p=0.6$. As $r$ increases from below the connectedness length to above the connectedness length, the form of the distribution changes from the power law distribution of Eq. (41) to a distribution with a pronounced peak, a characteristic of homogeneous systems. In Fig. 10b, in order to eliminate the finitesize effect, we select $L=r+2$ so that the distribution $P(t \mid r)$ does not have a power-law regime, even for small $r$. In this case, as shown in Fig. 10 c, the fractal dimension of the minimal traveling time crosses over from $d_{\mathrm{tm}}=1.33$ to $d_{\mathrm{tm}}=2.0$, characteristic of a homogeneous system 28,27. The same considerations that we use to derive the behavior of the mean and variance of the shortest path can be applied to the mean and variance of the minimal time. At the moment of breakthrough, i.e., when the first tracer particle reaches the second well, the part of the system filled with tracer particles consists of $n_{b}=(r / \xi)^{d}$ independent blobs, each of which having a certain number of bonds $\left(t_{\min }\right)_{b}$ with an average $\left\langle\left(t_{\min }\right)_{b}\right\rangle=\xi^{d_{\mathrm{tm}}}$ and a variance $\sigma_{b}^{2}=\xi^{2 d_{\mathrm{tm}}}$. Thus the average minimal time for the entire system scales as

$$
\left\langle t_{\mathrm{min}}\right\rangle=n_{b} \cdot \xi^{d_{\mathrm{tm}}}=r^{d} \xi^{d_{\mathrm{tm}}-d}
$$

with a variance

$$
\sigma^{2}=n_{b} \xi^{2 d_{\mathrm{tm}}}=r^{d} \xi^{2 d_{\mathrm{tm}}-d} .
$$

The scaling plot (Fig. 10 d) of $\left\langle t_{\min }\right\rangle$ versus $\left|p-p_{c}\right|$ shows good agreement with the theoretical prediction of Eq. (49)

$$
\begin{aligned}
\frac{\left\langle t_{\min }\right\rangle}{r^{d}} & =\left(p-p_{c}\right)^{\left(d-d_{\mathrm{tm}}\right) \nu} \\
& =\left(p-p_{c}\right)^{0.89} \quad[d=2] .
\end{aligned}
$$

The graph of $\sigma$ versus $r$ (see Fig. 10 e) shows linear behavior of $\sigma$ versus $r$ in agreement with Eq. (50). Equation (50) also predicts that the slope of this linear dependence decays as

$$
\left|p-p_{c}\right|^{-\left[d_{\mathrm{tm}}-(d / 2)\right] \nu}=\left|p-p_{c}\right|^{-0.42} \quad[d=2] .
$$

However the measured slope has very small variation with $\left|p-p_{c}\right|$ which is beyond the accuracy of our data points. 
As mentioned above, the minimal traveling time is the sum of the inverse local velocities over the fastest path where the fastest path is statistically identical to the shortest path. While the velocity distribution has been studied extensively 35,36, because the velocities along the path are correlated, the relation between the minimum traveling time distribution and the local velocity distribution is an open challenge for further research.

The analysis for three dimensions is completely analogous to that for two dimensions. Our results are shown in Figs. 11 and 12 and the scaling parameters found are included in Table I.

\section{Fastest Path}

We observe that the path which takes minimal time is not always the shortest path. However analysis of the distributions of $\ell_{\min }$ yields parameters identical to those for the distribution of the shortest paths between points separated by distance $r$ studied in detail in Ref. [15]. Thus, statistically, the path which takes the shortest time is one of the paths of shortest length.

In many transport problems, the characteristic time $t^{*}$ scales with the characteristic length $\ell^{*}$ with a power law,

$$
t^{*} \sim\left(\ell^{*}\right)^{z}
$$

Since $t^{*}$ scales as $r^{d_{t}}$ and $\ell^{*}$ scales as $r^{d_{\text {min }}}$,

$$
z=d_{t} / d_{\min }
$$

Since $t_{\min }$ and $\ell_{\min }$ are strongly correlated, the distributions $P\left(\ell_{\min }\right)$ and $P\left(t_{\min }\right)$ satisfy

$$
P\left(\ell_{\min }\right) d \ell_{\min }=P\left(t_{\min }\right) d t_{\min }
$$

Combining Eqs. (53)-(55) and the equations for the respective distributions, we obtain a scaling relation between exponents,

$$
\left(g_{\ell_{\min }}-1\right) d_{\ell_{\min }}=\left(g_{\mathrm{tm}}-1\right) d_{\mathrm{tm}} .
$$

These scaling relations are well satisfied by the set of scaling exponents given in Table I.

\section{CONCLUSIONS}

By modeling porous media by bond percolation and using concepts of percolation theory, we study the flow of fluid in porous media in 2 and 3 dimensions between two "wells" separated by Euclidean distance $r$. We investigate the distribution function of the shortest path connecting the two sites, and propose a scaling Ansatz that accounts for the dependence of this distribution (i) on $L$, the size of the system, and (ii) on $p$, the bond occupancy probability. The finite size of the system, $L$ corresponds to the size of the oil field and the bond occupancy probability, $p$ is related to the good sand to impermeable rock ratio of the porous media. We confirm by extensive simulations that the Ansatz holds for $d=2,3$, and we calculate the relevant scaling parameters.

In order to understand the properties of the flow of oil displaced by fluid or gas, we study the dynamics of flow on percolation clusters. We study two dynamical quantities: the minimal traveling time and the length of the path corresponding to the minimal traveling time. Because of the approximate parallel between the shortest path and the minimal traveling time of flow, the study of the shortest path is the first step in understanding the properties of the oil fields. In particular, a scaling Ansatz for these dynamical quantities includes the effect of finite system size and off-critical bond occupation probability. We find that the scaling form for the distribution functions for these dynamical quantities for $d=2,3$ is similar to, but not identical to, that for the shortest path. In addition to calculating the relevant distribution functions and scaling relations, we also calculate a number of new exponents (see Table I).

\section{ACKNOWLEDGMENTS}

We thank BP Amoco for financial support, and M. Barthélémy, A. Coniglio, J. Koplik, S. Redner, and R. M. Ziff for helpful discussions.

\section{APPENDIX A: SAMPLING ALL SIZE CLUSTERS VERSUS SAMPLING ONLY INFINITE CLUSTERS}

Since $P^{\prime}(\ell \mid r)$ is the distribution of shortest paths between point in clusters of all sizes (not just the infinite cluster), we can write

$$
P^{\prime}(\ell \mid r)=\int_{0}^{\infty} P(\ell \mid r, M) P(M) d M,
$$

where $P(\ell \mid r, M)$ is the distribution of shortest paths in clusters of mass $M$.

The size $L$ of a cluster of mass $M$ is of the order $M^{1 / d_{f}}$ and the shortest path between two points in the cluster is larger than $L^{d_{\text {min }}}$ drops off exponentially, so $P(\ell \mid r, M)$ will effectively be zero when $M$ is less than $\ell^{d_{f} / d_{\min }}$. On the other hand, when $M$ is greater than this value, $P(\ell \mid r, M)$ will be the same distribution as the distribution for the case when the two points are in an infinite cluster. This can be taken into account by replacing $P(\ell \mid r, M)$ with $P(\ell \mid r)$ and replacing the zero lower limit of the integral by $\ell^{d_{f}} / d_{\min }$. Since $P(M) \sim M^{-\tau}$, where $\tau=d / d_{f}$, we have

$$
\begin{aligned}
P^{\prime}(\ell \mid r) & =\int_{\ell^{d_{f} / d_{\min }}}^{\infty} P(\ell \mid r) M^{-d / d_{f}} d M \\
& =P(\ell \mid r) \ell^{\left(d_{f}-d\right) / d_{\min }},
\end{aligned}
$$


from which follows

$$
g_{\ell}^{\prime}=g_{\ell}+\frac{d-d_{f}}{d_{\min }}=g_{\ell}+\left(d-d_{f}\right) \tilde{\nu} .
$$

This can be generalized for any quantity, $x$, with fractal dimension $d_{x}$ and with distributions

$$
P(x \mid r)=\frac{1}{r^{d_{x}}}\left(\frac{x}{r^{d_{x}}}\right)^{-g_{x}}
$$

and

$$
P^{\prime}(x \mid r)=\frac{1}{r^{d_{x}}}\left(\frac{x}{r^{d_{x}}}\right)^{-g_{x}^{\prime}}
$$

where "infinite clusters" and all finite-sized clusters, respectively, are sampled. This generalization results in

$$
g_{x}^{\prime}=g_{x}+\frac{d-d_{f}}{d_{x}} .
$$

[1] S. R. Broadbent and J. M Hammersley, Proc. Camb. Phil. Soc. 53, 629 (1957).

[2] D. Stauffer and A. Aharony, Introduction to Percolation Theory (Taylor \& Francis, Philadelphia, 1994).

[3] S. Havlin and D. Ben-Avraham, Adv. Phys. 36, 695 (1987).

[4] Fractals and disordered systems, edited by A. Bunde and S. Havlin (Springer-Verlag, New York, 1996).

[5] M. Sahimi, Rev. Mod. Phys. 65, 1393 (1993).

[6] M. Sahimi, Applications of Percolation Theory (Taylor \& Francis, London, 1994).

[7] The Fractal Approach to Heterogeneous Chemistry: Surfaces, Colloids, Polymers, edited by D. Avnir (John Wiley \& Sons, Chichester, 1989).

[8] E.T. Gawlinski and H. E. Stanley, J. Phys. A 14, L291 (1981).

[9] A. Geiger and H. E. Stanley, Phys. Rev. Lett. 49, 1895 (1982).

[10] P. G. de Gennes, Scaling Concepts in Polymer Physics (Cornell University Press, Ithaca, 1979).

[11] J. M. Ziman, Models of Disorder (Cambridge University, Cambridge, 1979).
[12] V. Ambegaokar, B. I. Halperin, and J. S. Langer, Phys. Rev. B 4, 2612 (1971).

[13] A. J. Katz and A. H. Thompson, Phys. Rev. B 34, 8179 (1986); J. Geophys. Res. B 92, 599 (1987).

[14] J. S. Andrade Jr., D. A. Street, Y. Shibusa, S. Havlin, and H. E. Stanley, Phys. Rev. E 55, 772 (1997).

[15] N. V. Dokholyan, Y. Lee, S. V. Buldyrev, S. Havlin, P. R. King and H. E. Stanley, J. Stat. Phys. 93, 603 (1998); N. V. Dokholyan, S. V. Buldyrev, S. Havlin, P. R. King, Y. Lee, and H. E. Stanley, Physica A 266, 53 (1999).

[16] Y. Lee, J. S. Andrade, S. V. Buldyrev, N. Dokholyan, S. Havlin, P. R. King, G. Paul, and H. E. Stanley, Phys. Rev. E 60, 3425 (1999).

[17] P. R. King, in North Sea Oil and Gas Reservoirs III, edited by A. T. Buller et al. (Graham and Trotman, London, 1990).

[18] Z. Alexandrowicz, Phys. Lett. A 80, 284 (1980).

[19] R. Pike and H. E. Stanley, J. Phys. A: Math. Gen. 14, L169 (1981).

[20] H. J. Herrmann and H. E. Stanley, J. Phys. A: Math. Gen. 21, L829 (1988).

[21] P. Grassberger, J. Phys. A: Math. Gen. 25, 5475 (1992).

[22] P. Ray, J. Phys. A: Math. Gen. 18, L657 (1985).

[23] M. Barma and P. Ray, Phys. Rev. B 34, 3403 (1986).

[24] A. U. Neumann and S. Havlin, J. Stat. Phys. 52, 203 (1988).

[25] R. M. Ziff, J. Phys. A 32, L457 (1999).

[26] P. L. Leath, Phys. Rev. B 14, 5046 (1976).

[27] P. King, J. Andrade, N. V. Dokholyan, S. V. Buldyrev, S. Havlin, Y. Lee, and H. E. Stanley, Physica A 266, 107 (1999).

[28] J. Koplik, S. Redner, and E. J. Hinch, Phys. Rev. E 50, 4650 (1994).

[29] P. G. Saffman, J. Fluid Mech. 6, 321 (1959).

[30] J. Koplik, S. Redner, and D. Wilkinson, Phys. Rev. A 37, 2619 (1988).

[31] J.-C. Bacri et al., in Hydrodynamics of Dispersed Media, edited by J. P. Hulin, A. M. Cazabat, E. Guyon, and F. Carmona (Elsevier, North-Holland, 1990), p. 249.

[32] M. Sahimi, Flow and Transport in Porous Media and Fractured Rock (VCH, Boston, 1995).

[33] F. A. L. Dullien, Porous Media - Fluid Transport and Pore Structure (Academic Press, New York, 1979).

[34] H. J. Herrmann and H. E. Stanley, Phys. Rev. Lett. 53, 1121 (1984).

[35] L. de Arcangelis, S. Redner, and A. Coniglio, Phys. Rev. B 31, 4725 (1985).

[36] M. Barthélémy, S. V. Buldyrev, S. Havlin, and H. E. Stanley, Phys. Rev. E 61, 3283 (2000). 
Table I. Summary of exponents and coefficients in scaling form

$$
P(x \mid r) \sim\left(1 / r^{d_{x}}\right)\left(x / r^{d_{x}}\right)^{-g_{x}} f_{1}\left(x / r^{d_{x}}\right) f_{2}\left(x / L^{d_{x}}\right) f_{3}\left(x / \xi^{d_{x}}\right)
$$

where $f_{1}(y)=\exp \left(-a_{x} y^{-\phi_{x}}\right), f_{2}(y)=\exp \left(-b_{x} y^{\psi_{x}}\right), f_{3}(y)=\exp \left(-c_{x} y\right)$. Here $x$ denotes one of the quantities, $\ell$ or $t_{\min }$. The notation N/A means Not Applicable (since no theoretical value exists), while the notation (+/-) indicates above or below $p_{c}$.

(a) $d=2$ results

\begin{tabular}{|c||c|c||c|c||}
\hline \multirow{2}{*}{$\begin{array}{c}x \\
\text { exponent }\end{array}$} & \multicolumn{2}{c||}{$\ell$} & \multicolumn{2}{c||}{$t_{\min }$} \\
\cline { 2 - 5 } & sim. & theory & sim. & theory \\
\hline$d_{x}$ & $1.13 \pm 0.01$ & $N / A$ & $1.33 \pm 0.05$ & $N / A$ \\
\hline$g_{x}^{\prime}$ & $2.14 \pm 0.02$ & 2.11 & $2.0 \pm 0.1$ & $N / A$ \\
\hline$a_{x}$ & 0.5 & $N / A$ & 1.1 & $N / A$ \\
\hline$\phi_{x}$ & $7.3 \pm 0.5$ & $1 /\left(d_{x}-1\right)=7.69$ & 3.0 & 3.0 \\
\hline$b_{x}$ & 3.5 & $N / A$ & 5.0 & $N / A$ \\
\hline$\psi_{x}$ & $4.0 \pm 0.5$ & $N / A$ & 3.0 & $N / A$ \\
\hline$c_{x}$ & $2.4(-), 3.7(+)$ & $N / A$ & $1.6(-), 2.6(+)$ & $N / A$ \\
\hline
\end{tabular}

(b) $d=3$ results

\begin{tabular}{|c||c|c||c|c||}
\hline \multirow{2}{*}{\multicolumn{1}{c||}{$\begin{array}{c}x \\
\text { exponent }\end{array}$}} & \multicolumn{3}{c||}{$\ell$} & \multicolumn{2}{c||}{$t_{\min }$} \\
\cline { 2 - 5 } & sim. & theory & sim. & theory \\
\hline$d_{x}$ & $1.39 \pm 0.05$ & $N / A$ & $1.45 \pm 0.1$ & $N / A$ \\
\hline$g_{x}^{\prime}$ & $2.3 \pm 0.1$ & 2.23 & $2.1 \pm 0.1$ & $N / A$ \\
\hline$a_{x}$ & 1.4 & $N / A$ & 2.5 & $N / A$ \\
\hline$\phi_{x}$ & $2.1 \pm 0.5$ & $1 /\left(d_{x}-1\right)=2.56$ & 1.6 & 2.0 \\
\hline$b_{x}$ & 2.0 & $N / A$ & 2.3 & $N / A$ \\
\hline$\psi_{x}$ & $2.5 \pm 0.5$ & $N / A$ & 2.0 & $N / A$ \\
\hline$c_{x}$ & $3.1(-)$ & $N / A$ & $2.9(-)$ & $N / A$ \\
\hline
\end{tabular}



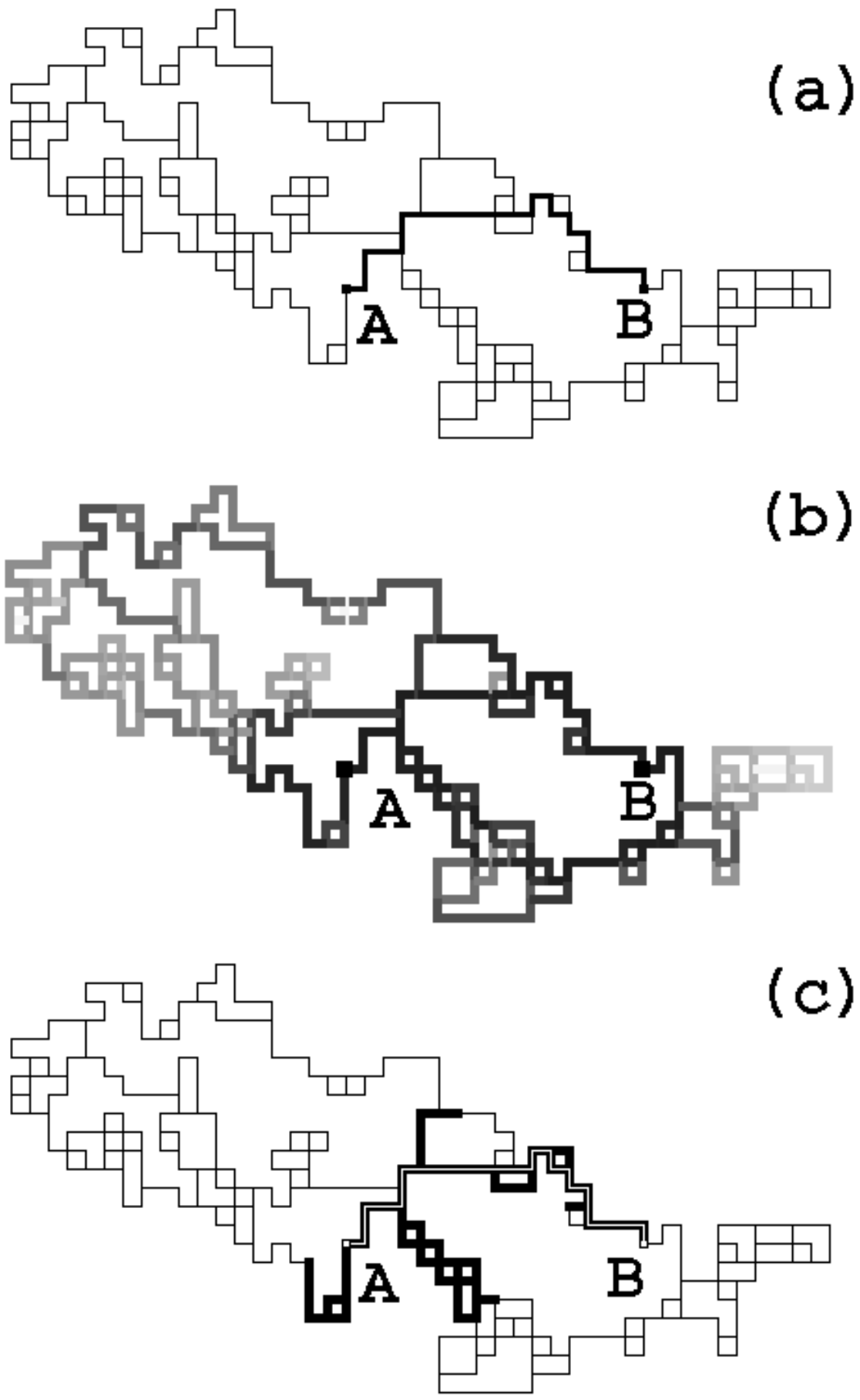

FIG. 1. An example of (a) percolation backbone (thin lines) and shortest path (thick line) corresponding to the flow between points $A$ and $B$-note that the lines here represent not microscopic pores but rather sand bodies whose size are of the order of tens of meters; (b) magnitudes of currents on cluster backbone are depicted in gray scale with the lightest areas corresponding to the smallest currents and the darkest to the largest currents; and (c) time evolution of the flow between points $A$ and $B$ on a two-dimensional percolation cluster. Thick lines denote bonds reached by tracer particles at time $t_{\text {min }}$. The double line between $A$ and $B$ denotes the "fastest path" between these two points. 

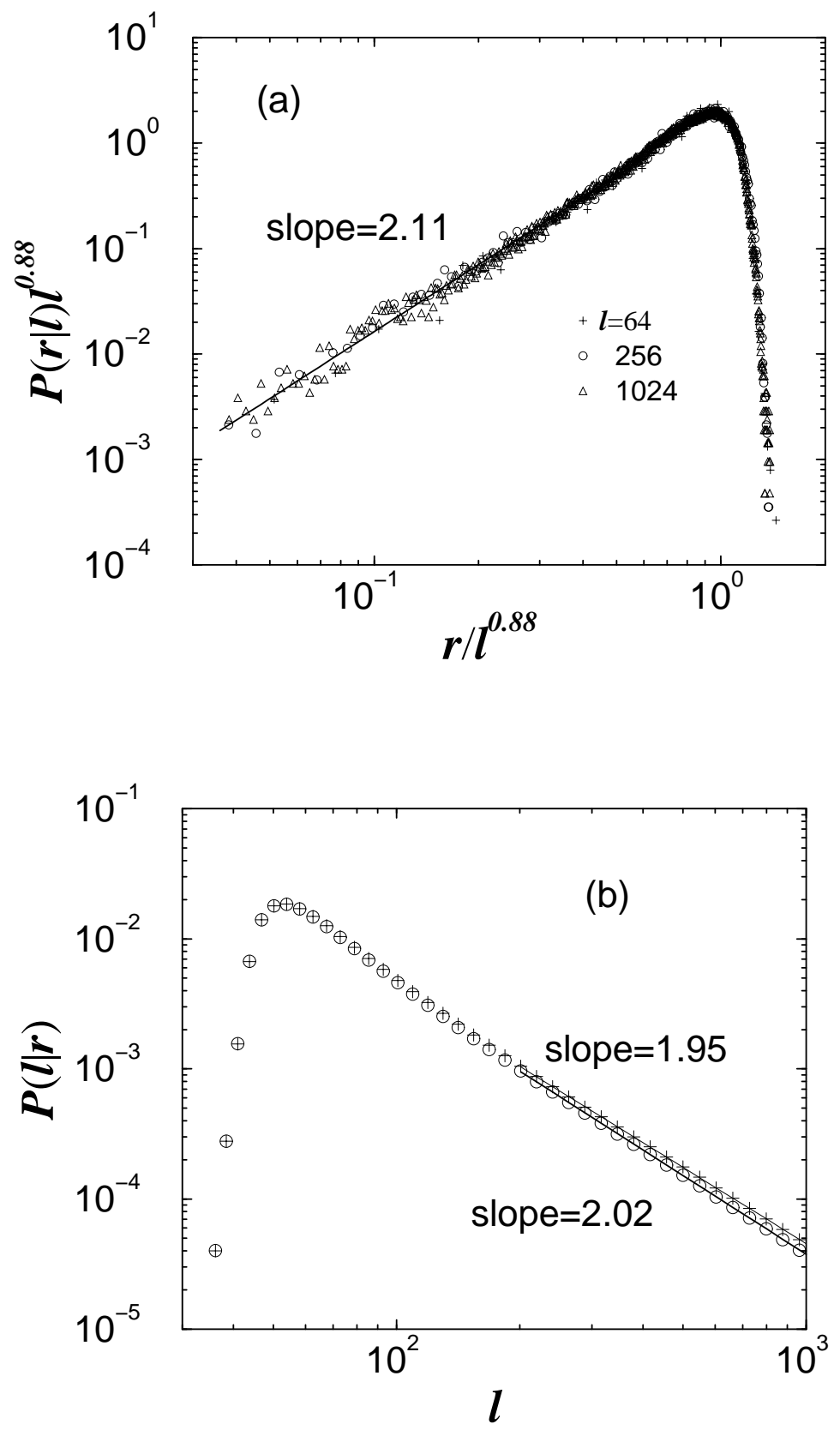

FIG. 2. (a) Log-log plot of $\ell^{\nu} P(r \mid \ell)$ for two-dimensional percolation at the percolation threshold for $\ell=64(\bigcirc)$, $256(\square)$ and $1024(\triangle)$. The best data collapse is obtained with $\nu=0.88$ and the slope of the power regime is 2.11 . (b) Log-log plot of $P(\ell \mid r)(+)$ and $P^{\prime}(\ell \mid r)(\bigcirc)$ for two-dimensional percolation at criticality and for the system size $L=1024$ and the distance between wells is $r=32$. The power-law regime of $P^{\prime}(\ell \mid r)$ has slope $g_{\ell}^{\prime}=2.02$ (thick solid line), while that of $P(\ell \mid r)$ has slope $g_{\ell}=1.95$ (thin solid line). The purpose of this figure is to illustrate the difference between $g_{\ell}$ and $g_{\ell}^{\prime}$. The values shown here are lower than those predicted by Ziff 25] because we have not included corrections to scaling in our determination of these quantities. 

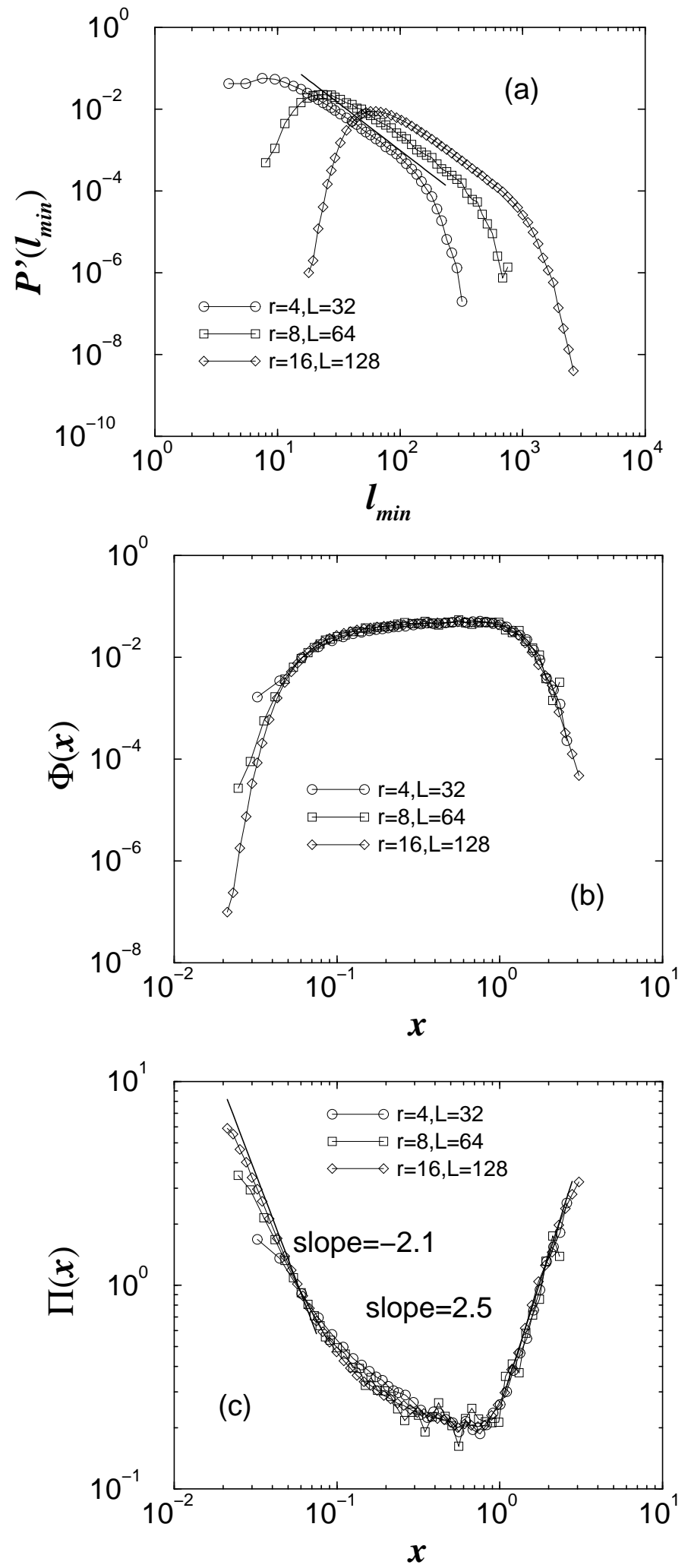
FIG. 3. For $d=3$, (a) log-log plot of $P^{\prime}(\ell \mid r)$ at criticality $\left(p=p_{c} \approx 0.2488\right)$ and for different sets of parameters: $(r, L)=(2,32),(4,64),(8,128)$. The straight line regime has slope $g_{\ell}^{\prime}=2.3$. (b) Log-log plot of rescaled probability $\Phi(x) \equiv P^{\prime}(\ell \mid r) x^{g_{\ell}^{\prime}} r^{d} \min$ against rescaled length $x \equiv \ell / r^{d} \min$ using the values, $g_{\ell}^{\prime}=2.3$ and $d_{\text {min }}=1.39$. The curves are flat in the center because $f_{2}(x)$ is a stretched exponential (see Eq. (25)). (c) Log-log plot of transformed probability $\Pi(x)=\log _{10}[A / \Phi(x)]$ versus $x=\ell / r^{d}$ min. The slopes of the solid lines give the power of the stretched exponential function $f_{1}$ and $f_{2}$ in Eq. 25). Using the parameter $A=0.08$, the slopes give $\phi \approx 2.1$ for the lower cut-off and $\psi \approx 2.5$ for the upper cut-off. 

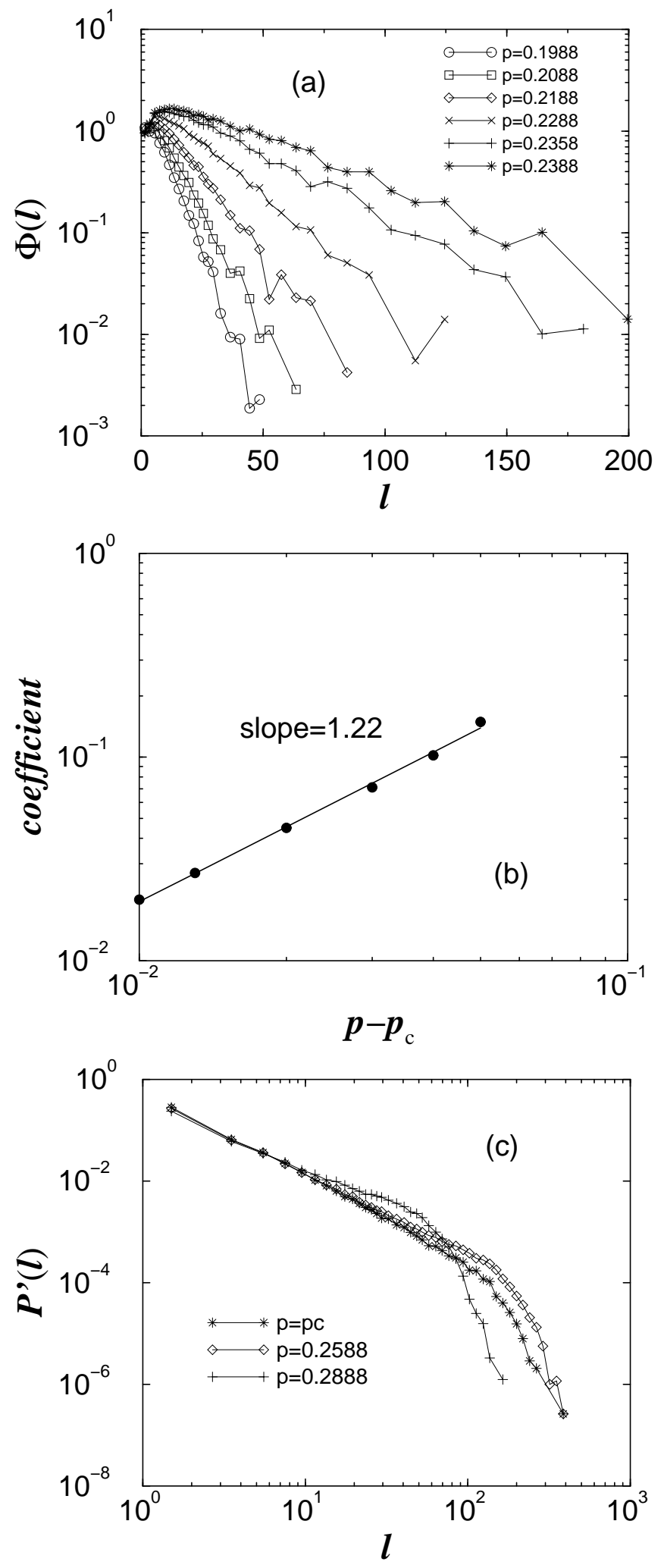
FIG. 4. For $d=3$, (a) semi-logarithmic plot of transformed probability $\Phi(\ell)$ (see Eq. (25)) versus $\ell$ shows pure exponential behavior of $f_{3}$. (b) The slope of the log-log plot of the coefficient in exponential function $f_{3}$ as a function of $\left|p-p_{c}\right|$ gives the value $\nu d_{\text {min }} \approx 1.22$ for $p<p_{c}$. (c) $P^{\prime}(\ell)$ for $p>p_{c}$. Note that it is only for $p \geq 0.2788$ that the large $\ell$ behavior is determined by the fact that the system is not at criticality. 

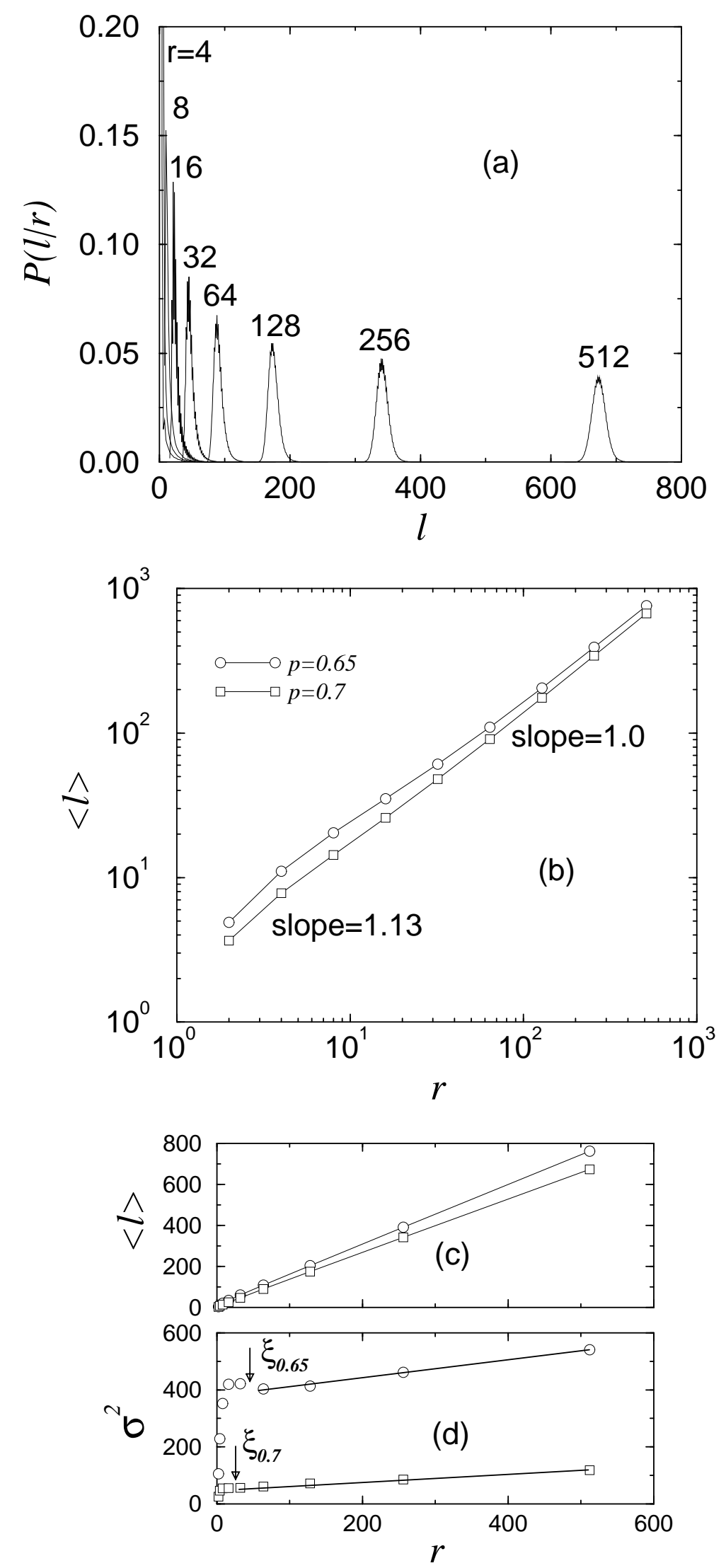
FIG. 5. (a) Distributions of $P(\ell \mid r)$ for $r=4,8,16,32,64,128,256,512$ and for $p=0.7$. To reduce the lattice effects, data is obtained for the pairs of wells on the $x$-axis. (Note that for this case, where $r>\xi$, the distributions $P^{\prime}(\ell \mid r)$ and $P(\ell \mid r)$ are essentially the same since all the clusters span the lattice.) The distributions converge for large $r$ to a Gaussian with mean $\langle\ell\rangle$ shown in parts (b,c) and variance $\sigma^{2}=\left\langle\ell^{2}\right\rangle-\langle\ell\rangle^{2}$, shown in part (d) as functions of $r$ for $p=0.65(\bigcirc)$ and $p=0.7(\square)$. (b) Log-log plot of $\langle\ell\rangle$ versus $r$. Note the crossover from power law behavior with exponent $d_{\text {min }}=1.13$ to linear behavior with exponent 1.0. (c) Same as (b) on linear scale. The slopes of linear the fits $k(p)$ are 1.45 for $p=0.65$ and 1.30 for $p=0.7$. This yields $k(p) \sim\left(p-p_{c}\right)^{-0.17}$ in good agreement with equation Eq.(30). (d) The dependence of $\sigma^{2}$ versus $r$. According to Eq.(29), the dependence becomes linear only for $r>\xi \sim\left(p-p_{c}\right)^{-\nu}$, indicated on the graph. The slopes of linear fits $k(p)$ are 0.33 for $p=0.65$ and 0.12 for $p=0.7$. This gives $k(p) \sim\left(p-p_{c}\right)^{-1.6}$ in good agreement with equation Eq.(31). 

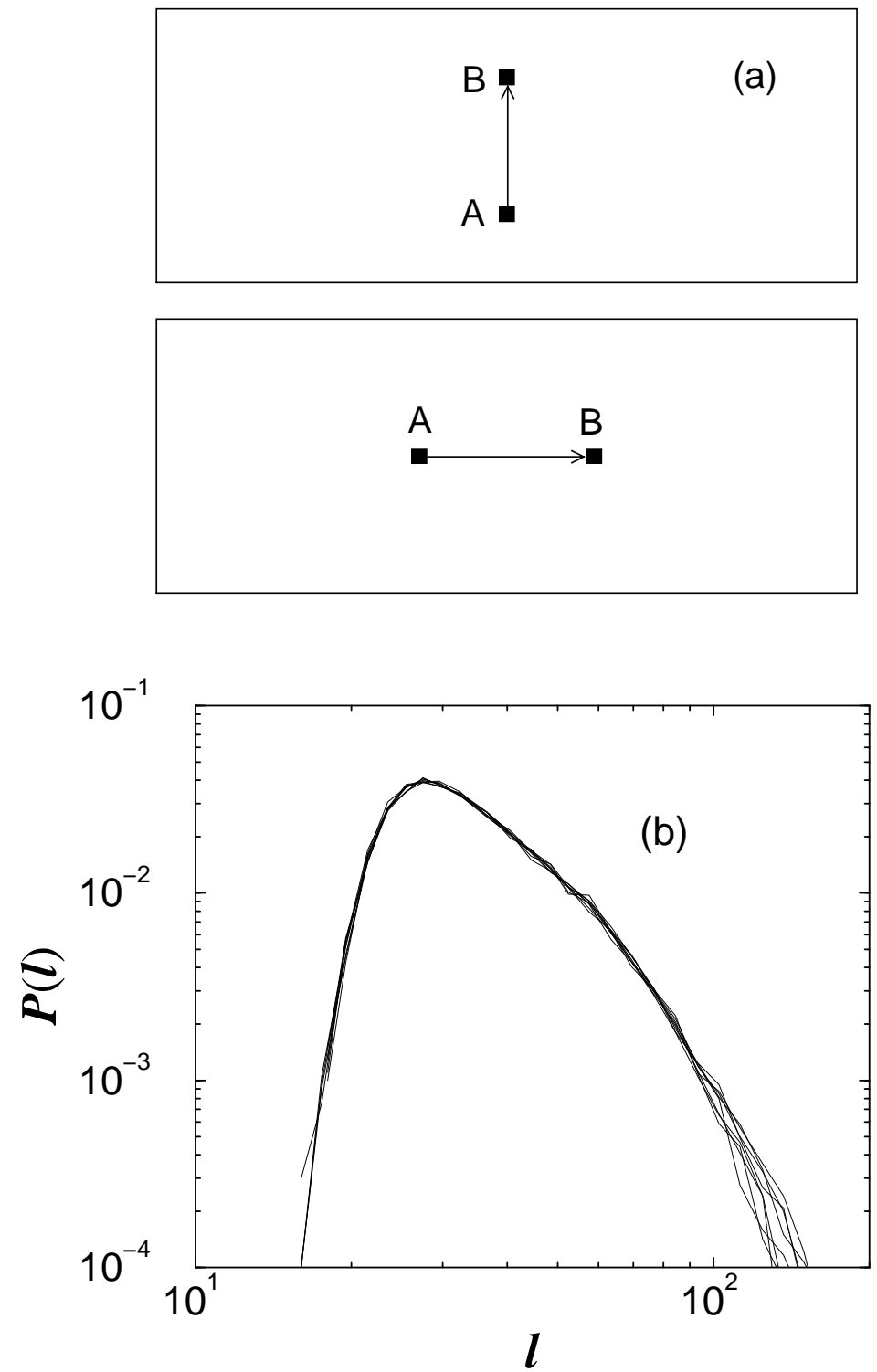

FIG. 6. (a) Two configurations with rectangular boundaries are studied. (b) For $r=4$, different system sizes $\left(L_{x}, L_{y}\right)=(32,128),(32,256),(32,512),(32,1024),(128,32),(256,32),(512,32)$ and $(1024,32)$ are studied and all the distributions of minimal path are collapsed into each other. 


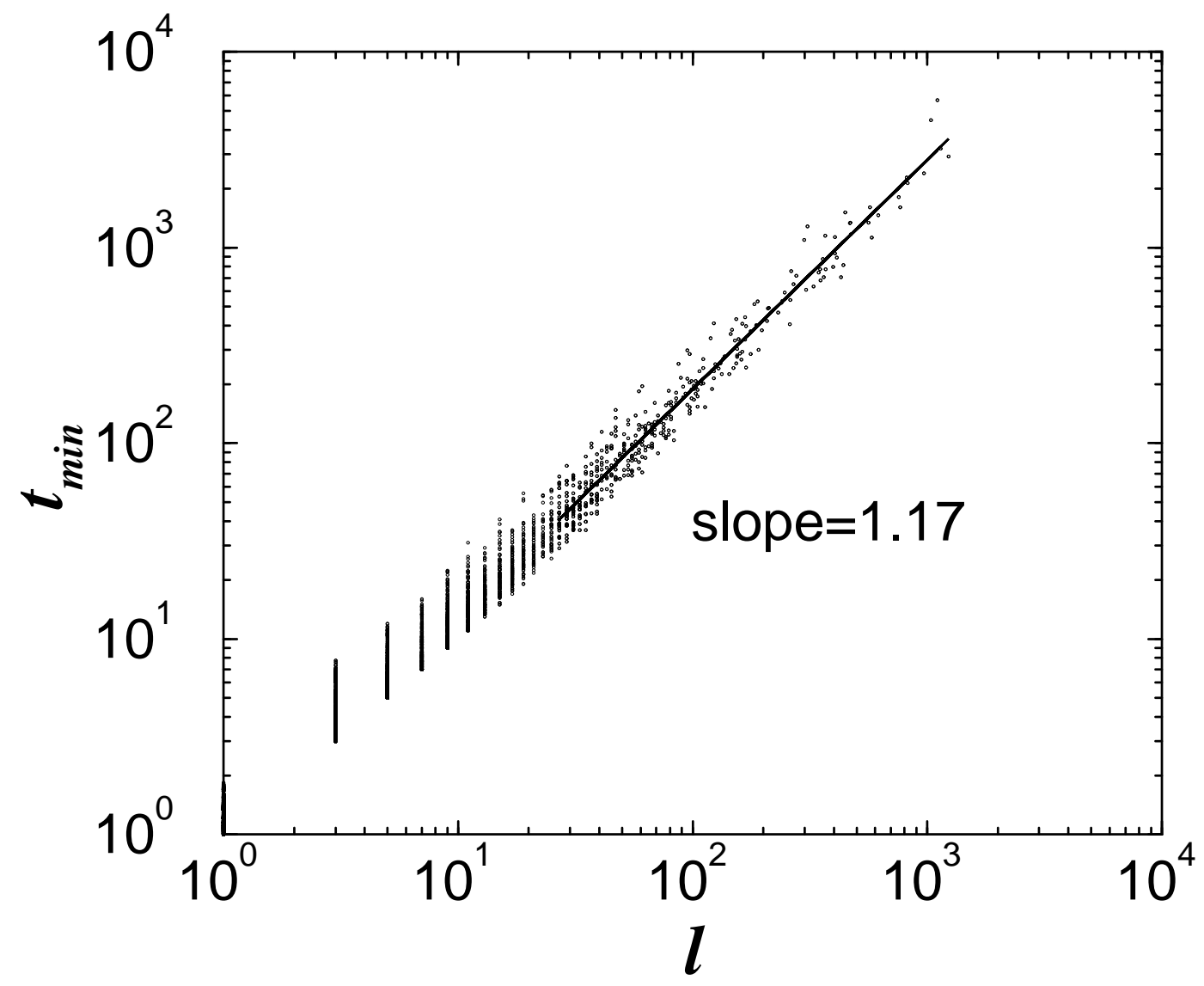

FIG. 7. For $d=2$, scatter plot of the minimal traveling time $t_{\text {min }}$ versus minimal length $\ell$ and for a fixed well separation $r=1$. Note the strong correlation between $t_{\min }$ and $\ell$. The slope of the tail of the scatter plot is 1.17 yielding a value of $d_{t m}=1.17 \cdot d_{\min }=1.32$, consistent with our result in Table I. 

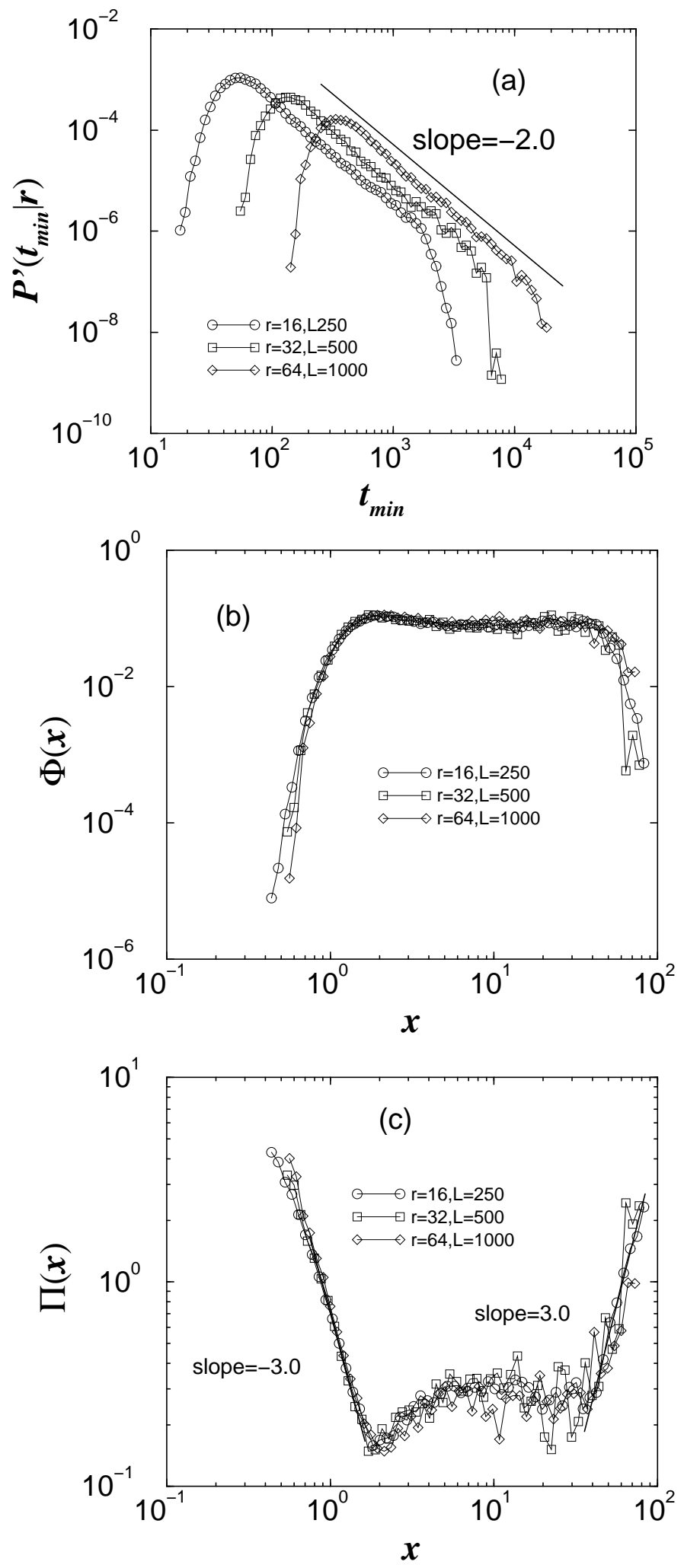
FIG. 8. For $d=2$, (a) $\log$ - $\log$ plot of $P^{\prime}(t \mid r)$ for $p=p_{c}=0.5$ and for different sets of parameters, $(r, L)=(16,250),(32,500),(64,1000)$. The straight line regime has slope $g_{t}^{\prime}=2.0$. (b) Log-log plot of rescaled probability $\Phi(x) \equiv P^{\prime}\left(t_{\min } \mid r\right) x^{g_{t}^{\prime}} r^{d_{t}}$ against rescaled length $x=t_{\mathrm{min}} / r^{d_{t}}$ using the values, $g_{t}^{\prime}=2.0$ and $d_{t}=1.33$. The curves are flat in the

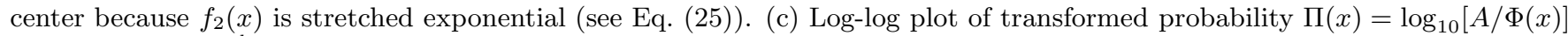
versus $x=t_{\mathrm{min}} / r^{d_{t}}$. The slopes of the solid lines give the power of the stretched exponential function $f_{1}$ and $f_{2}$ in Eq. (25). Using the parameter $A=0.14$, the slopes give $\phi \approx 3.0$ for the lower cut-off and $\psi \approx 3.0$ for the upper cut-off. 

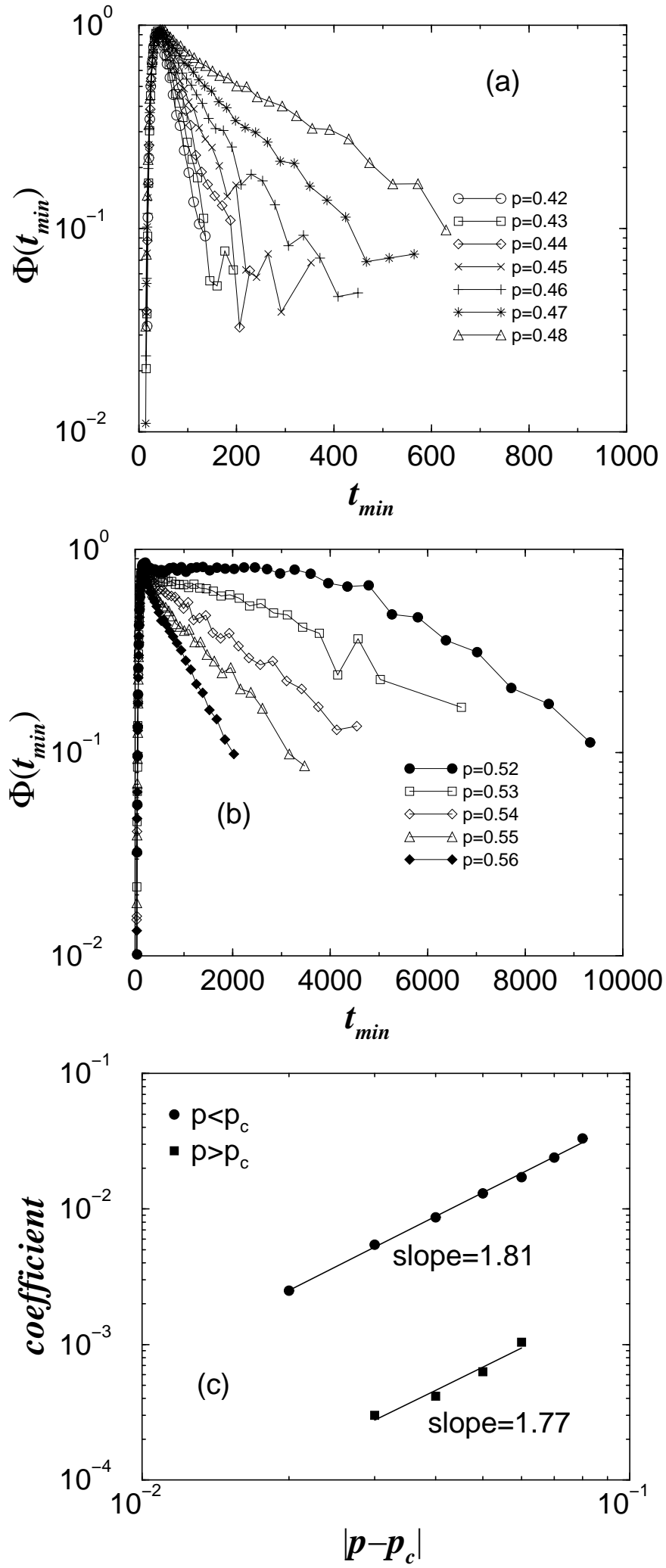
FIG. 9. For $d=2$, (a) semi-logarithmic plot of transformed probability $\Phi\left(t_{\min } / r^{d_{t}}\right)$ versus $t_{\min }$ for $f_{3}$ for $p=0.42,0.43,0.44,0.45,0.46,0.47,0.48$ below criticality. (b) Same for $p=0.52,0.53,0.54,0.55,0.56$ above criticality. (c) The slope of the $\log -\log$ plot of the coefficient in exponential function $f_{3}$ as a function of $\left|p-p_{c}\right|$ gives the value $\nu d_{t} \approx 1.77$ for $p>p_{c}$ and 1.81 for $p<p_{c}$. 

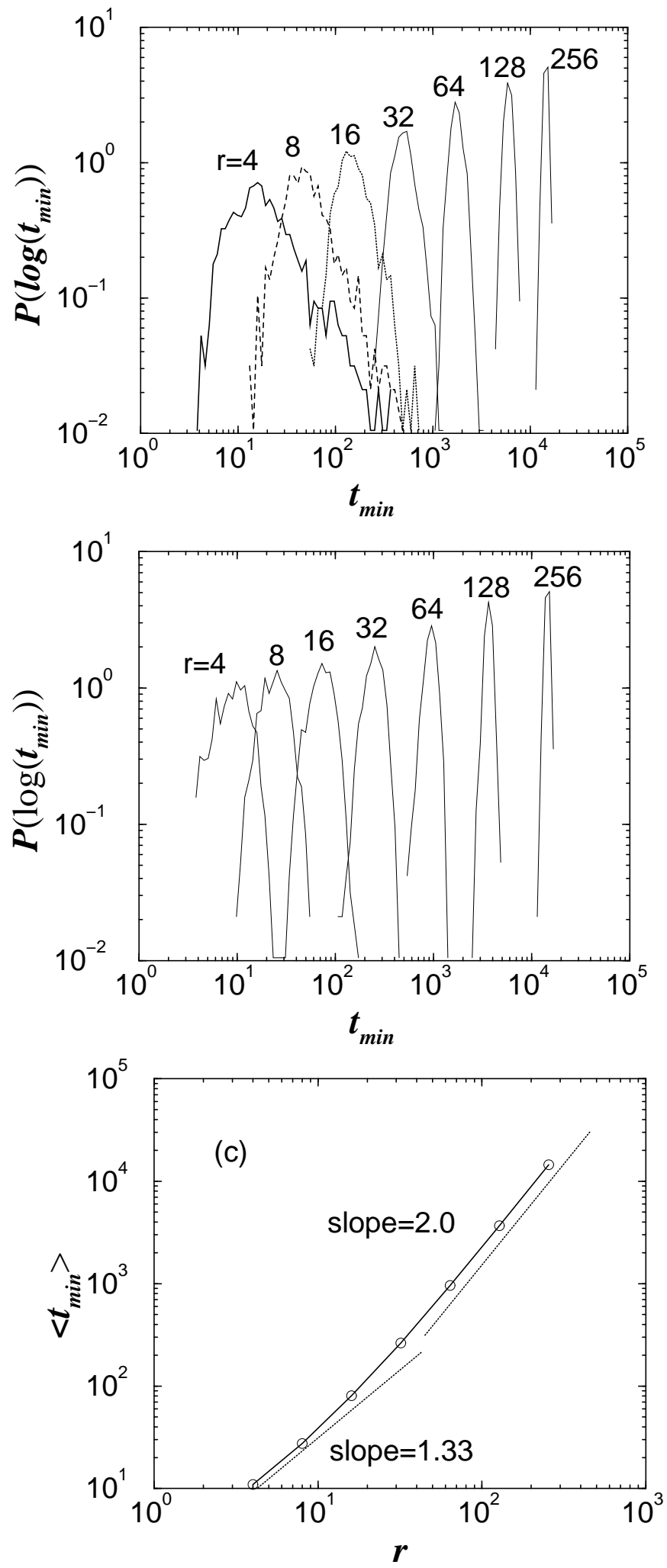

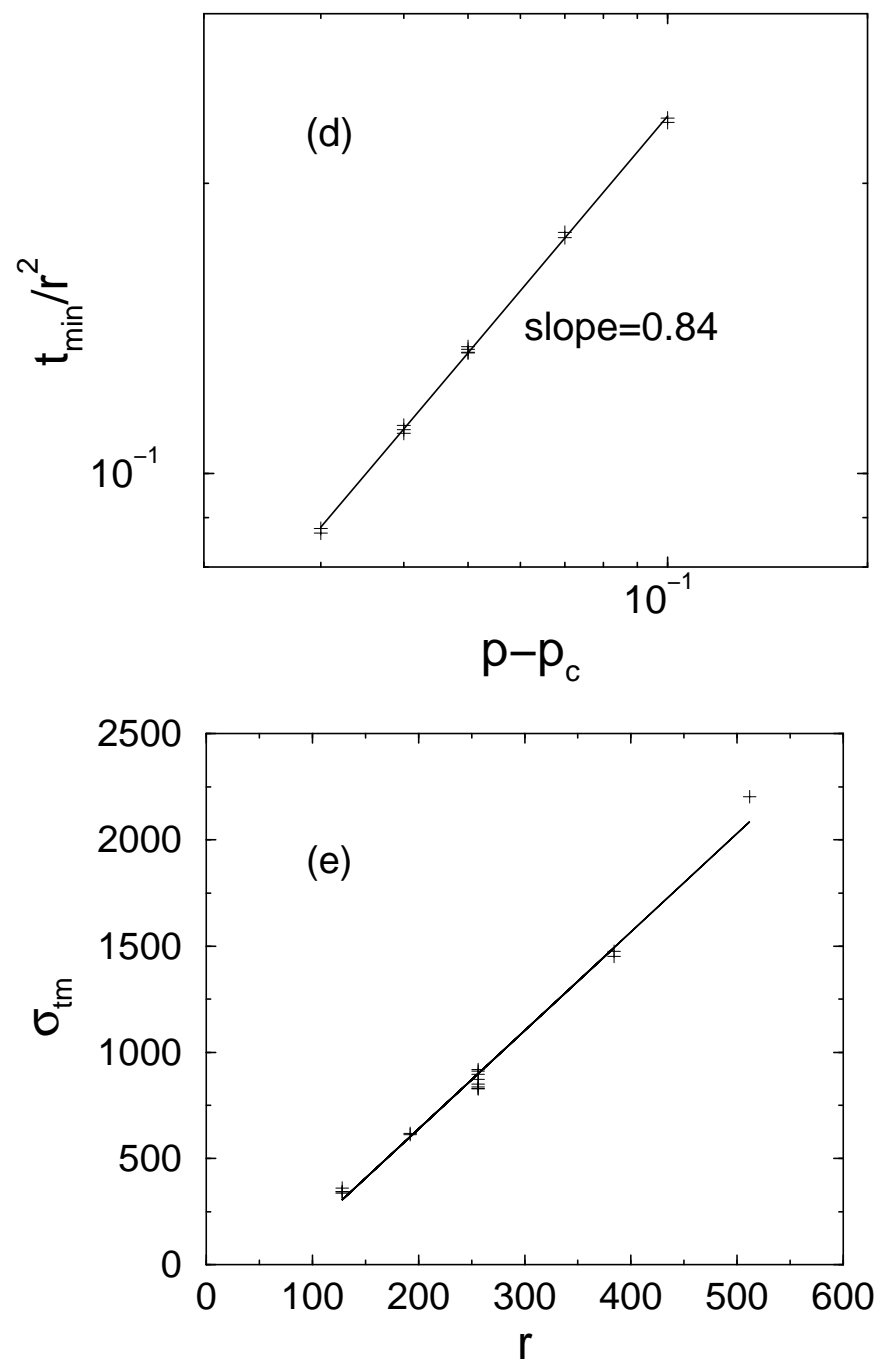

FIG. 10. (a) Log-log plot of $P\left(t_{\min } \mid r\right)$ for $p=0.6$ and for $r=4,8,16,32,64,128,256$ and $L=258$. The distributions for large $r$ converge to Gaussians with mean $\left\langle t_{\min }\right\rangle$ and variance $\sigma^{2}$. (b) $\log -\log$ plot of $P\left(t_{\min } \mid r\right)$ for $p=0.6, r=4,8,16,32,64,128,256$ and $L=r+2$. (Note that for this case, where $r>\xi$, the distributions $P^{\prime}\left(t_{\min } \mid r\right)$ and $P\left(t_{\min } \mid r\right)$ are essentially the same since all the clusters span the lattice.) (c) Log-log plots of $\left\langle t_{\min }\right\rangle$ versus $r$ for $p=0.6$ and $L=r+2$. (d) Log-log plot of the scaled average minimal traveling time, $\left\langle t_{\min }\right\rangle / r^{2}$, versus $p-p_{c}$ for $r=128,192,256,384,512$ and $L=r+2$. Note that in all cases $r \gg \xi$. The slope of the line, 0.84, is in good agreement with the theoretical prediction, 0.89. (e) The behavior of the width, $\sigma$, of the distributions of the traveling time versus $r$ for $p=0.53,0.54,0.55,0.57$ and 0.6 . The graph shows approximately linear dependence of $\sigma$ on $r$. The variation of the slope with $p-p_{c}$ is within the error bars of the data. 

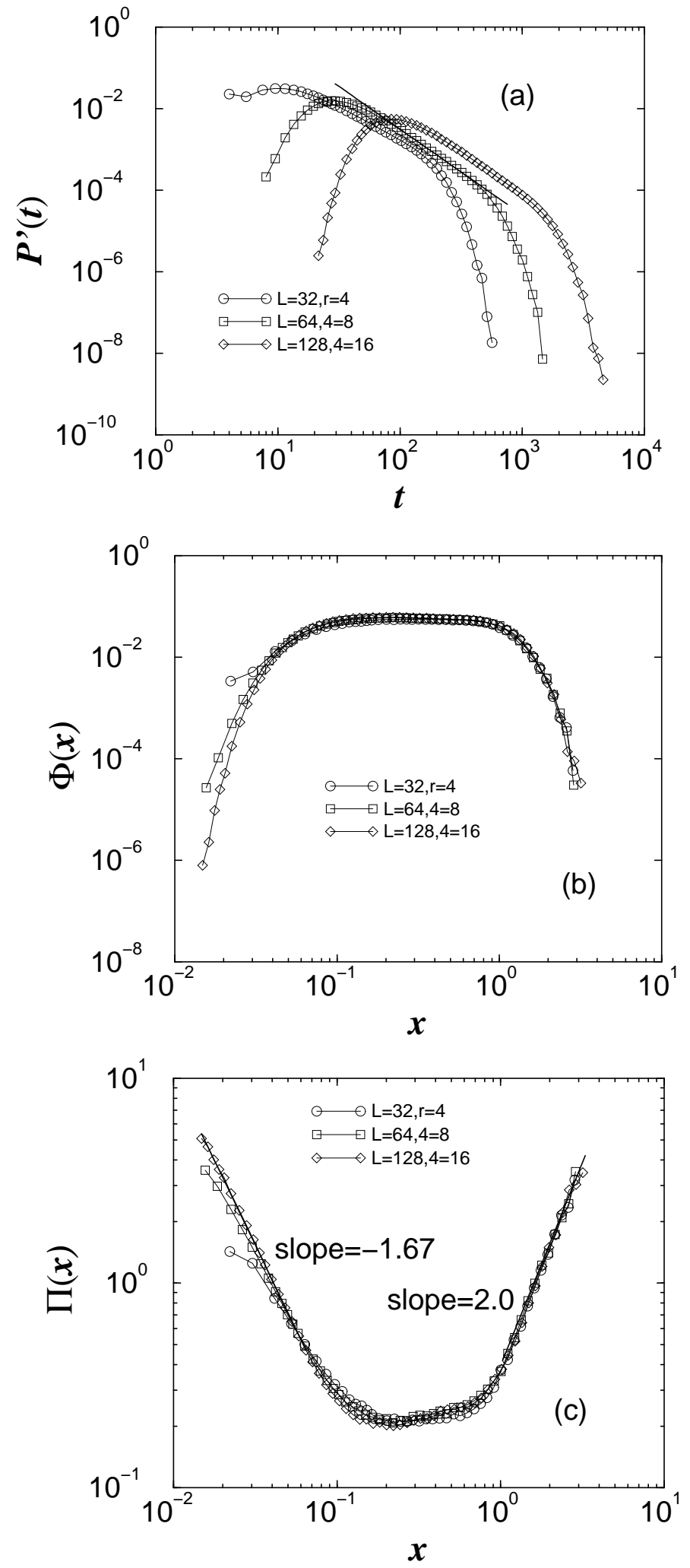
FIG. 11. For $d=3$,(a) log-log plot of $P^{\prime}(t \mid r)$ for $p=p_{c}=0.2488$ and for different sets of parameters, $(r, L)=(4,32),(8,64),(16,128)$. The power-law regime has slope $g_{t}^{\prime}=2.1$. (b) Log-log plot of rescaled probability $\Phi(x) \equiv P^{\prime}\left(t_{\min } \mid r\right) x^{g_{t}^{\prime}} r^{d_{t}}$ against rescaled length $x=t_{\mathrm{min}} / r^{d_{t}}$ using the values, $g_{t}^{\prime}=2.1$ and $d_{t}=1.45$. The curves are flat in the

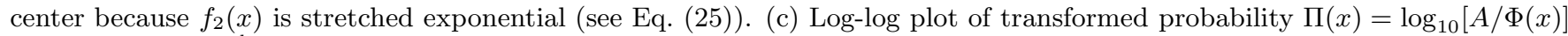
versus $x=t_{\mathrm{min}} / r^{d_{t}}$. The slopes of the solid lines give the power of the stretched exponential function $f_{1}$ and $f_{2}$ in Eq. (25). Using the parameter $A=0.08$, the slopes give $\phi \approx 1.6$ for the lower cut-off and $\psi \approx 2.0$ for the upper cut-off. 

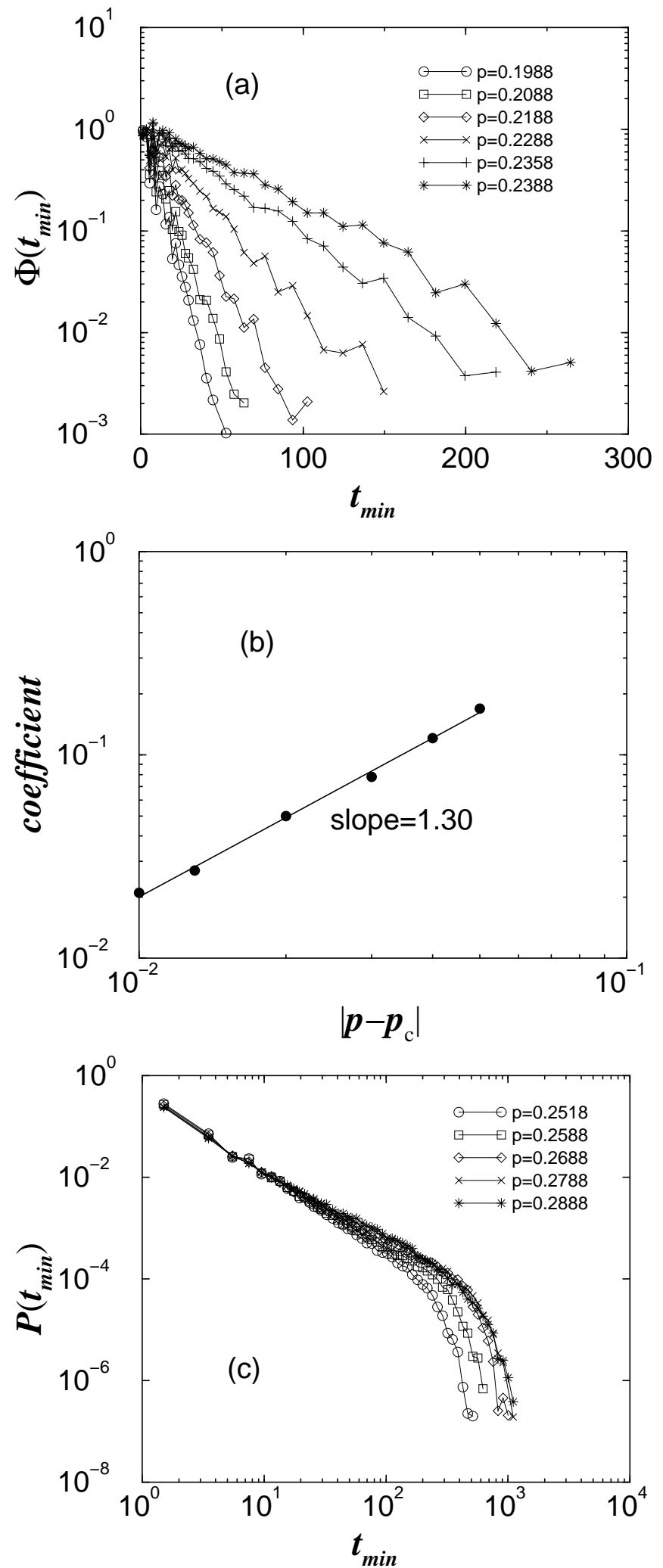
FIG. 12. For $d=3$, (a) semi-logarithmic plot of transformed probability $\Phi\left(t_{\text {min }}\right)$ versus $t_{\text {min }}$ below critical point for $p=0.1988,0.2088,0.2188,0.2288 .0 .2358,0.2388$ shows pure exponential behavior of $f_{3}$. (b) The slope of the log-log plot of the coefficient in exponential function $f_{3}$ as a function of $\left|p-p_{c}\right|$ gives the value $\nu d_{t} \approx 1.30$ for $p<p_{c}$. (c) $P\left(t_{\text {min }}\right)$ for $p>p_{c}$. Note that for the values of $p$ simulated, the large $t_{\min }$ behavior is determined by the finite size of the system-not $f_{3}$. 